\title{
1 Functional analysis of a common BAG3 allele 2 associated with protection from heart failure
}

3 Juan A Perez-Bermejo ${ }^{1}$, Luke $M$ Judge ${ }^{1,2}$, Christina L Jensen ${ }^{1}$, Kenneth $\mathrm{Wu}^{1}$,

4 Annie Truong ${ }^{1}$, Jaclyn $\mathrm{J} \mathrm{Ho}^{3}$, Matthew Carter $^{1}$, Wendy $\vee$ Runyon ${ }^{1}$, Robyn M

5 Kaake ${ }^{1,4,5}$, Ernst Pulido ${ }^{1}$, Hannah L Watry ${ }^{1}$, Mohammad A Mandegar ${ }^{3}$, Danielle L

6 Swaney $^{1,4,5}$, Po-Lin So ${ }^{1}$, Nevan J Krogan ${ }^{1,4,5 *}$, Bruce R Conklin ${ }^{1,5,6,7 *}$

$8{ }^{1}$ Gladstone Institutes, San Francisco, CA, United States

9 UCSF Departments of ${ }^{2}$ Pediatrics, ${ }^{5}$ Cellular Molecular Pharmacology and ${ }^{6}$ Medicine,

10 San Francisco, CA, United States

$11{ }^{3}$ Tenaya Therapeutics, South San Francisco , CA, United States

$12{ }^{4}$ UCSF Quantitative Biosciences Institute (QBI), San Francisco, CA, United States

$13{ }^{7}$ Innovative Genomics Institute, Berkeley, CA, United States 


\section{Abstract}

16 Multiple genetic association studies have correlated a common allelic block linked to the

17 BAG3 gene with a decreased incidence of heart failure, but the molecular mechanism

18 for such protection remains elusive. One of the variants in this allele block is coding,

19 changing cysteine to arginine at position 151 of BAG3 (rs2234962-BAG3 ${ }^{\mathrm{C} 151 \mathrm{R}}$ ). Here,

20 we use induced pluripotent stem cells (iPSC) to test if the BAG3 ${ }^{\mathrm{C} 151 \mathrm{R}}$ variant alters

21 protein and cellular function in human cardiac myocytes. Quantitative protein interaction

22 network analysis identified specific changes in BAG3 ${ }^{\mathrm{C} 151 \mathrm{R}}$ protein interaction partners in

23 cardiomyocytes but not in iPSCs or an immortalized cell line. Knockdown of BAG3

24 interacting factors in cardiomyocytes followed by myofibrillar analysis revealed that

$25 \mathrm{BAG}^{\mathrm{C} 151 \mathrm{R}}$ associates more strongly with proteins involved in the maintenance of

26 myofibrillar integrity. Finally, we demonstrate that cardiomyocytes expressing the

27 BAG3 ${ }^{C 151 R}$ variant have improved response to proteotoxic stress in an allele dose-

28 dependent manner. This study suggests that the BAG3 ${ }^{C 151 R}$ variant increases

29 cardiomyocyte protection from stress by enhancing the recruitment of factors critical to

30 the maintenance of myofibril integrity, hinting that this variant could be responsible for

31 the cardioprotective effect of the haplotype block. By revealing specific changes in

32 preferential binding partners of the $\mathrm{BAG} 3^{\mathrm{C} 151 \mathrm{R}}$ protein variant, we also identify potential

33 targets for the development of novel cardioprotective therapies. 


\section{$\underline{\text { Introduction }}$}

Heart failure is a major cause of mortality, with increasing incidence each year ${ }^{1}$.

37 One of the main causes of non-ischemic heart failure is dilated cardiomyopathy (DCM),

38 which has a strong genetic component ${ }^{1-3}$. Although many genetic association studies

39 have identified risk loci associated with DCM, the translation of these discoveries into

40 therapeutic strategies remains challenging. This is partly due to a lack of mechanistic

41 insights at the molecular level, making it difficult to progress from correlation to

42 causality. In addition, most of the genetic variants identified in genetic association

43 studies are rare and disease-causing, so the mechanistic information is applicable to

44 only a small subset of patients. On the contrary, variants associated with a decreased

45 risk of disease (protective) have the potential to be utilized for the development of

46 therapeutic strategies that are applicable to a much broader range of subjects ${ }^{4}$.

47 Recently, multiple large genome wide association studies have implicated a common

48 haplotype block, which overlaps with the BAG3 (Bcl-2 associated athanogene 3) gene,

49 with an apparent decreased incidence of DCM or with improved cardiac ejection

50 fraction ${ }^{5-13}$ (for a summary of these studies, see Table 1). Despite the strong genetic

51 association, the molecular basis of the apparent protective effect remains unknown. A

52 mechanistic understanding of this protective allele could aid in the development of novel

53 cardioprotective therapies.

A combination of clinical case reports and large genetic association studies have implicated BAG3 loss-of-function variants with DCM ${ }^{5,7,14-20}$. BAG3 was first described as an HSP70 co-chaperone, while successive studies have found it to be a multifunctional

58 hub for a diverse set of cellular processes, mostly centered around protein quality

59 control and selective autophagy ${ }^{21-23}$. In cardiac and skeletal muscle, a functioning

60 protein quality control network is essential to dynamically maintain myofibrillar structures

61 through constant cycles of contraction ${ }^{24}$. Accordingly, BAG3 impairment results in heart

62 and skeletal muscle pathology ${ }^{25-28}$. These studies indicate the BAG3 protein has an

63 essential role in muscle, particularly in cardiomyocytes, which lack replicative potential.

64 In particular, BAG3 binding to the HSP70 chaperones is essential for cardiac health, as

65 indicated by multiple pathogenic mutations that reduce this interaction ${ }^{14,15,17,18,29}$. 
66 Interestingly, the cardioprotective BAG3 allele block contains one coding variant,

67 rs2234962, which leads to the BAG3 ${ }^{\mathrm{C} 151 \mathrm{R}}$ amino acid transition. However, the C151R

68 change is located distant from the HSP70 binding domain, in an intrinsically disordered

69 region with no known function. Based on these observations, we hypothesize that the

70 BAG3 ${ }^{\mathrm{C} 151 \mathrm{R}}$ could induce a novel functional effect in the BAG3 protein and be

71 responsible for the effect of the cardioprotective haplotype block.

73 Recent studies have demonstrated that induced pluripotent stem cell (iPSC)

74 modeling can be used to elucidate the functional effects of disease risk variants

75 identified in genetic association studies ${ }^{30-33}$. Here, we have leveraged the power of

76 iPSC technology and genome editing to examine the impact of the C151R aminoacid

77 change in the function of the BAG3 protein, and on the response of iPSC-derived

78 cardiomyocytes to stress. Our findings confirm that the $B A G 3^{C 151 R}$ variant has a

79 protective effect in vitro, and could be responsible for the effect of the cardioprotective

80 haplotype block. We also discovered $B A G 3^{C 151 R}$-associated alterations in protein

81 interactions that are cardiomyocyte specific and hint at novel mechanisms for

82 cardioprotection. 
84 Table 1.- Summary of genome wide association studies supporting the putative

85 cardioprotective effect of the BAG3 haplotype block.

\begin{tabular}{|c|c|c|c|c|c|}
\hline Study & Phenotype & SNP* & MAF & \multicolumn{2}{|c|}{$\begin{array}{l}\text { Odds } \\
\text { Ratio }\end{array}$} \\
\hline \multicolumn{6}{|c|}{ DCM/Heart Failure studies } \\
\hline Villard et al, $2011^{5}$ & DCM & rs2234962 & 0.17 & 0.66 & 4.00E-12 \\
\hline Esslinger et al, $2017^{6}$ & DCM & rs2234962 & 0.19 & 0.62 & $1.70 \mathrm{E}-25$ \\
\hline $\begin{array}{l}\text { Aragam et al, } 2018 \\
(\text { UK Biobank })^{7}\end{array}$ & $\begin{array}{l}\text { Non Ischemic } \\
\text { Cardiomyopathy }\end{array}$ & rs2234962 & 0.22 & 0.77 & $3.55 \mathrm{E}-07$ \\
\hline $\begin{array}{l}\text { Aragam et al, } 2018 \\
(\text { BioVU })^{7}\end{array}$ & $\begin{array}{l}\text { Non Ischemic } \\
\text { Cardiomyopathy }\end{array}$ & rs2234962 & 0.21 & 0.67 & $3.12 \mathrm{E}-03$ \\
\hline $\begin{array}{l}\text { Aragam et al, } 2018 \\
(\text { GRADE })^{7}\end{array}$ & $\begin{array}{l}\text { Non Ischemic } \\
\text { Cardiomyopathy }\end{array}$ & rs2234962 & 0.21 & 0.72 & $1.00 \mathrm{E}-02$ \\
\hline Shah et al, $2020^{8}$ & Heart Failure & rs17617337 & 0.22 & 0.94 & 3.65E-09 \\
\hline Choquet et al, $2020^{9}$ & HF/Cardiomyopathy & rs17617337 & 0.2 & 0.9 & $2.90 \mathrm{E}-02$ \\
\hline Verweij et al, $2020^{10}$ & Heart Failure & rs2234962 & 0.21 & 0.71 & $6.69 \mathrm{E}-05$ \\
\hline de Denus et al, $2020^{11}$ & Idiopathic DCM & rs2234962 & 0.22 & 0.42 & $5.00 \mathrm{E}-04$ \\
\hline Garnier et al, $2021^{12}$ & DCM & rs61869036 & 0.18 & 0.67 & $5.60 \mathrm{E}-14$ \\
\hline \multicolumn{6}{|l|}{ LVEF/ECG studies } \\
\hline Aung et al, $2019^{13}$ & LVEF on non-HF patients & rs2234962 & \multicolumn{3}{|c|}{$\begin{array}{l}\text { Improved LVEF 0.5\%/allele } \\
(p-v a l=2.00 E-14)\end{array}$} \\
\hline Choquet et al, $2020^{9}$ & LVEF (all patients) & rs17617337 & \multicolumn{3}{|c|}{$\begin{array}{l}\text { Improved LVEF } 0.8 \% \text { /allele } \\
(p-v a l=8.24 E-17)\end{array}$} \\
\hline Verweij et al, $2020^{10}$ & $\begin{array}{l}\text { Electrocardiogram } \\
\text { (all patients) }\end{array}$ & rs2234962 & \multicolumn{3}{|c|}{$\begin{array}{l}\text { Change in Q-R phase } e^{* *} \\
(p-v a l=4.80 E-54)\end{array}$} \\
\hline
\end{tabular}

Abbreviations: MAF - Minor Allele Frequency; DCM - Dilated Cardiomyopathy; LVEF - Left Ventricular Ejection Fraction; HF - Heart Failure

$*:$ SNPs rs17617337, rs61869036 and rs2234962(BAG3 $\left.{ }^{\mathrm{C} 151 \mathrm{R}}\right)$ are in complete LD (r2>0.99)

${ }^{* *} \mathrm{Q}-\mathrm{R}$ phase of ECG corresponds to ventricular depolarization and contraction 
$\underline{\text { Results }}$

$89 B A G 3^{C 151 R}$ quantitatively alters the profile of BAG3 interaction partners in cardiomyocytes

The BAG3 allele block associated with reduced incidence of heart failure

92 comprises at least seven common variants in almost complete linkage disequilibrium

93 across an $\sim 18 \mathrm{~Kb}$ region, of which only the rs2234962 variant is exonic (Fig 1A; Fig

94 S1A). The block presents with an allele frequency of roughly $20 \%$ in European and

95 South Asian populations (Fig 1B; Fig S1B). The rs2234962 variant results in the

$96 \mathrm{BAG}^{\mathrm{C} 151 \mathrm{R}}$ amino acid change, which is located in a region of the protein with no

97 annotated domain or function (Fig 1C; Fig S1C-D). Since BAG3 is a scaffold protein

98 with a wide range of binding partners, we reasoned that if this variant indeed alters

99 BAG3 function, it would likely do so via alterations in the profile of protein interactions.

100 Presumably, these changes would be different from those caused by a pathogenic

101 variant such as the BAG3 ${ }^{\mathrm{E} 455 \mathrm{~K}}$, which lies in a highly conserved region of the protein

102 (Fig S1E) and has been shown to disrupt protein interactions in the mouse

103 heart $^{20,27}$ (Fig 1C).

104

To test this hypothesis, we first performed affinity purification coupled to mass spectrometry (APMS) to characterize the stable protein interaction partners of a set of

107 BAG3 variants overexpressed in a non-muscle immortalized cell line, HEK293T. We 108 observed a significant loss of co-precipitation partners (mostly HSP70 family members)

109 for the pathogenic BAG3 variants (Fig 1D; Fig S2; Table S1). This result validated our

110 approach, however no changes in protein interactions were observed for BAG3 ${ }^{\mathrm{C} 151 \mathrm{R}}$.

111 We postulated that to see a difference for this variant, performing these experiments in

112 a more relevant cell type, the cardiomyocyte, was necessary. We also reasoned that

113 since BAG3 protein levels are tightly regulated, it would be important to use

114 endogenous levels of expression to study interaction partners. We therefore generated

115 a series of isogenic induced pluripotent stem cell (IPSC) lines bearing the BAG3 ${ }^{\mathrm{C} 151 \mathrm{R}}$

116 variant, the pathogenic variant $B A G 3^{E 455 K}$, or the non-disease associated wild-type

117 variant (BAG3 ${ }^{W T}$ ), with a 3xFLAG epitope tag fused to the endogenous BAG3 gene (Fig

118 S3). The cell lines were differentiated into cardiomyocytes (iPS-CMs) and we used 
119 APMS to analyze the BAG3 protein complexes in both iPSCs and iPS-CMs. As

120 expected, we observed a difference in the significant stable partners of the BAG3 ${ }^{\mathrm{E} 455 \mathrm{~K}-}$

$1213 \times{ }^{3 x L A G}$ variant compared to BAG3 ${ }^{W T-3 x F L A G}$ in both iPSCs and IPS-CMs (Fig 1D). In

122 comparison to the HEK293T APMS results, we were also able to characterize both

123 increasing and decreasing protein interactions for the BAG3 151 R-3xFLAG variant, but only

124 in the iPS-CM background and not in undifferentiated iPSCs (Fig 1D). This highlights

125 the cell type specificity of the BAG3 ${ }^{\mathrm{C} 151 \mathrm{R}}$ variant function. In addition, we performed this

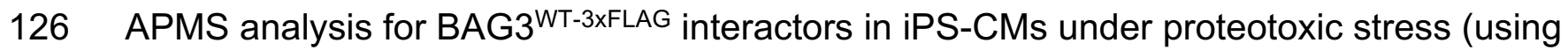

127 proteasome inhibitor bortezomib), and in an engineered inducible cell line

128 overexpressing BAG3 ${ }^{\mathrm{WT}-3 \times \mathrm{xLAG}}$ (Fig S4). This comprehensive characterization allowed us

129 to obtain a refined list of stable BAG3 interactors in a cardiomyocyte background (Fig

130 S5). This list included HSP70 chaperones and co-chaperones as well as other partners

131 such as small heat shock proteins, sarcomeric factors and actin-associated proteins. It

132 also showed a large difference in interactors identified when BAG3 was overexpressed,

133 highlighting the importance of performing functional analyses with proteins expressed at

134 endogenous levels (Fig S5D).

To quantify the changes in co-precipitation efficiencies of BAG3 interaction

137 partners across variants, we performed a quantitative targeted proteomics analysis on

138 the iPS-CM APMS samples. While the pathogenic variant BAG3 ${ }^{\mathrm{E} 455 \mathrm{~K}-3 \times F L A G}$ resulted in a

139 generalized loss of protein interactions compared to BAG3WT (mostly HSP70

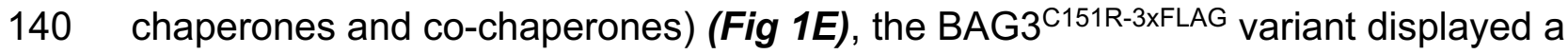

141 significant change in a different subset of binding partners (Fig 1F). In particular, there

142 was an increase in the interaction with actin-binding protein Filamin A, hippo pathway

143 kinase STK38, and E3 ubiquitin protein ligases DDB1 and TRIM21, while interaction

144 with small heat shock protein HSPB7 and co-chaperone DNAJB1 were decreased

145 compared to BAG3 ${ }^{W T}$.

Taken together, these results suggest that the putative protective variant

148 BAG3 ${ }^{\text {151R }}$ influences BAG3 protein function by modifying its cardiomyocyte-specific 149 profile of protein interactions. 
bioRxiv preprint doi: https://doi.org/10.1101/2021.10.06.463213; this version posted October 6, 2021. The copyright holder for this preprint (which was not certified by peer review) is the author/funder, who has granted bioRxiv a license to display the preprint in perpetuity. It is made available under aCC-BY-ND 4.0 International license.
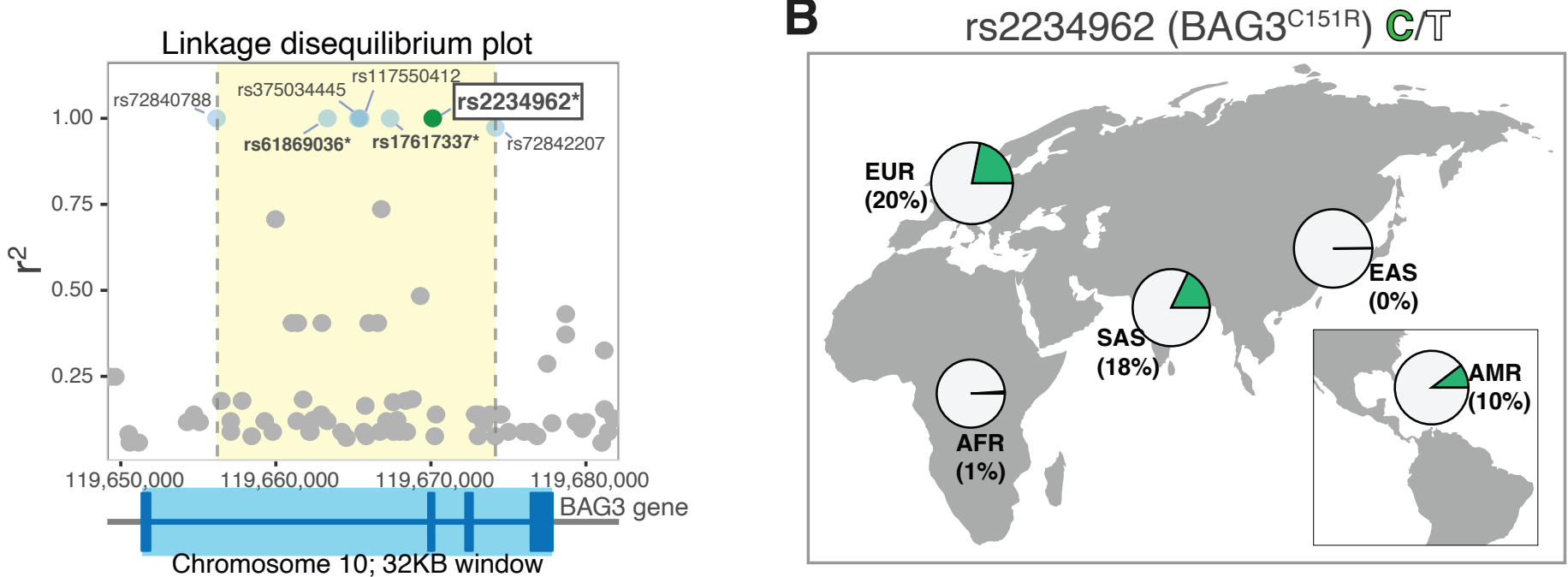

C
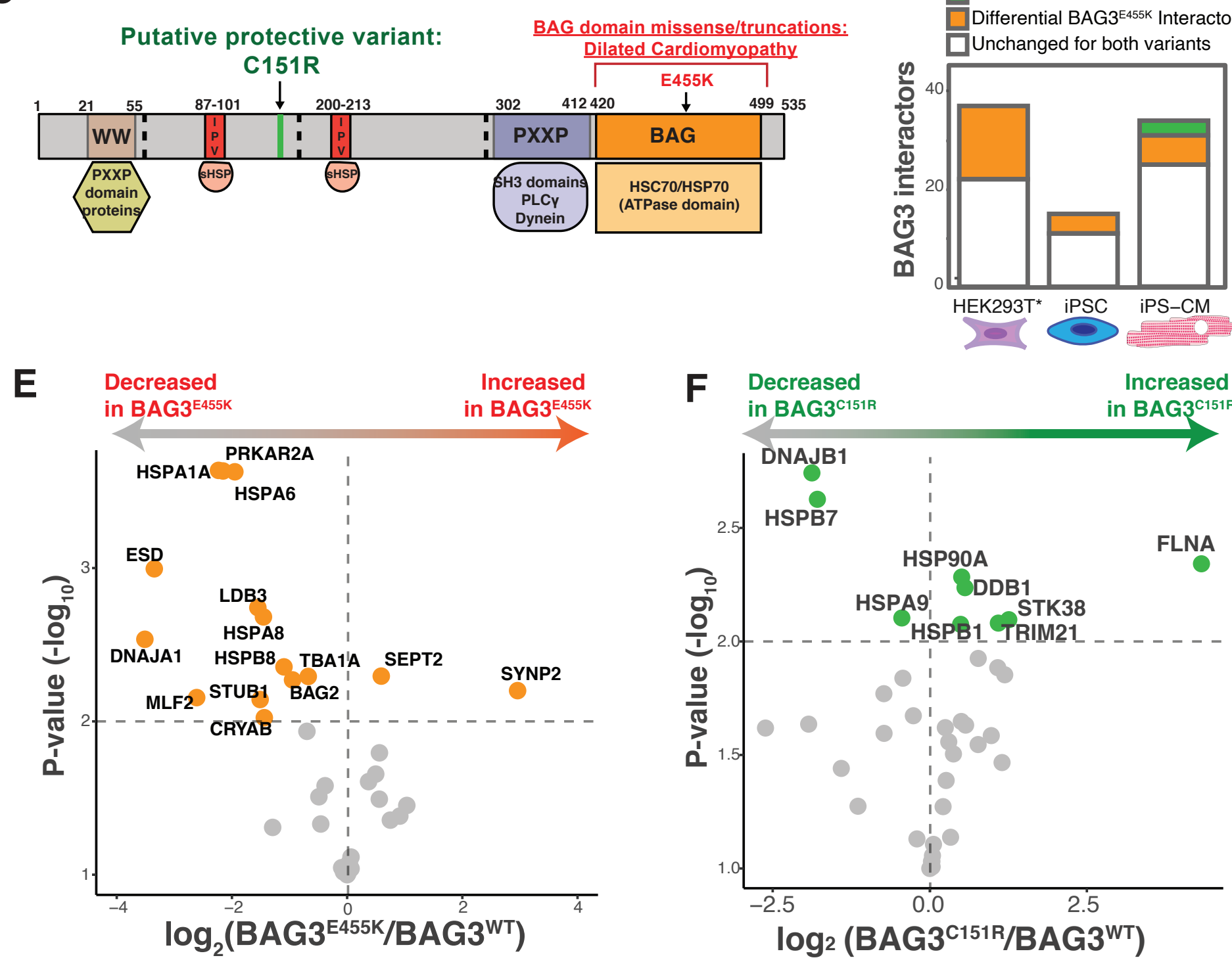


\section{Figure 1. The putative cardioprotective variant rs2234962-BAG3 ${ }^{\mathrm{C} 151 \mathrm{R}}$ results in a change} in the profile of BAG3 co-precipitation partners in a cardiomyocyte background. (A) Top: linkage disequilibrium plot centered around the rs2234962 variant (green) showing six other genetic variants (blue) in complete disequilibrium that form a haplotype block. *: Variants used in genetic association studies. Data for Central European population (CEU). Bottom: gene structure of the overlapping BAG3 gene (light blue: introns, dark blue: exons). (B) Allele frequencies across different super populations from the 1000 Genomes database. (AFR: African, AMR: Ad Mixed American, EAS: East Asian, EUR: European, SAS: South East Asian). (C) Schematic of BAG3 protein domain structure, highlighting some relevant interaction partners and indicating the location of the variants used in this study (arrows). Numbers indicate amino acid boundaries for annotated domains. Dashed lines indicate exon-exon boundaries. Areas of unknown function are colored in grey. (D-F) Interaction analyses for three different BAG3 variants $\left(B A G 3^{W T}, B A G 3^{C 151 R}, B A G 3^{E 455 K}\right.$ ). (D) Bar plot of the number of differential interactors (co-precipitation partners that interacted significantly more or less with BAG3 in either variant compared to BAG3 ${ }^{\text {WT }}$ ) identified using APMS in three different cell backgrounds - HEK293T cells (*: indicates BAG3 variant overexpression), undifferentiated human iPSC, and differentiated iPS-CM. Significantly different binding partners of BAG3 ${ }^{C 151 R}$ were only identified in the iPS-CM background. $N=4$. (E) Volcano plot depicting the intensity ratio of co-precipitation of each interactor with BAG3 ${ }^{\mathrm{E} 455 \mathrm{~K}-3 \times \mathrm{FLAG}}$ relative to $\mathrm{BAG} 3^{\mathrm{WT}-3 \times \mathrm{FLAG}}$ in $\mathrm{iPS}-\mathrm{CM}$. (F) Volcano plot depicting the intensity ratio of co-precipitation of each interactor with $B A G 3^{\text {C151R-3xFLAG }}$ relative to

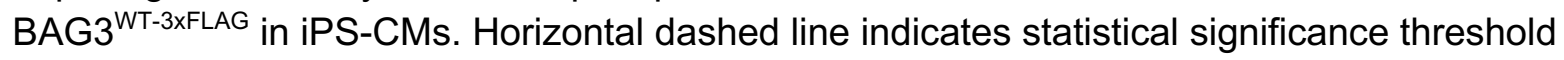
(adjusted $p$-value $<0.01$ ) and vertical dashed line indicates no ratio change. BAG3 ${ }^{\mathrm{C} 151 \mathrm{R}}$ results in a significant change of interactions that are different from those affected by the pathogenic variant. $N=4$. 


\section{$B A G 3^{C 151 R}$ engages factors involved in the maintenance of sarcomeric} homeostasis

Many stable BAG3 interaction partners identified here (such as those that significantly changed in the BAG3 ${ }^{\mathrm{C} 151 \mathrm{R}}$ background) have no known roles in cardiomyocyte function, particularly in the context of BAG3 mediated protein homeostasis. Cardiomyocytes are contractile cells and the structure and integrity of their myofibrils is closely associated to their functional output and pathological status.

157 Correspondingly, myofibrillar disarray is a hallmark of BAG3-related disease ${ }^{19,25,34}$. We 158 therefore hypothesized that some BAG3 partners might also play a role in the maintenance of sarcomeric integrity in iPS-CMs. To test this hypothesis in an unbiased manner, and with a focus on BAG3-specific features, we set up a pipeline for systematic iPS-CM gene knockdown followed by unbiased sarcomere structure analysis of microscopy images (Fig 2A). Images from different gene knockdowns were scored using a supervised learning classifier that ranked sarcomere staining images by similarity to a BAG3 knockdown phenotype or a control phenotype, generating a 'BAG3 sarcomere score'. Visual inspection of these two training conditions revealed that iPSCMs with a BAG3 knockdown were more likely to collapse (with completely aggregated myofibrils) and generally presented a lower myofibril density accompanied with increased sarcomere disarray and staining gaps (Fig 2B; Fig S6A-C). Our scoring scheme accurately classified BAG3 knockdown and control test images using only sarcomere staining images as input, and the obtained score correlated with BAG3 protein levels (Fig 2C, Fig S7A-C).

We then applied this phenotypic analysis pipeline to the knockdowns of all the 174 protein interactors identified in our BAG3 interactome in addition to some other high175 confidence BAG3-related proteins (Table S2). We found that knockdown of 9 of the 32 176 genes encoding BAG3 iPS-CM high confidence protein interactions (and 6 out of 19 177 genes from outside our APMS dataset) phenocopied BAG3 insufficiency (Fig 2D, Fig 178 S7D, Fig S8). None of these knockdowns significantly affected cardiomyocyte viability, 179 and only one (HSPB8, a well-known BAG3 partner in protein quality control ${ }^{26,35}$ ) 180 significantly decreased BAG3 protein levels (Fig S7E-F). Interestingly, knockdown of 
bioRxiv preprint doi: https://doi.org/10.1101/2021.10.06.463213; this version posted October 6,2021 . The copyright holder for this preprint

A (which was not certified by peer review) is the author/funder, who has granted bioRxiv a license to display the preprint in perpetuity. It is made available under ACC-BY-ND 4.0 International license.

Train automated image classification model

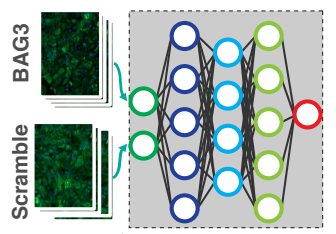

List of

BAG3-associated SiRNA knockdown Proteins on pure iPS-CM
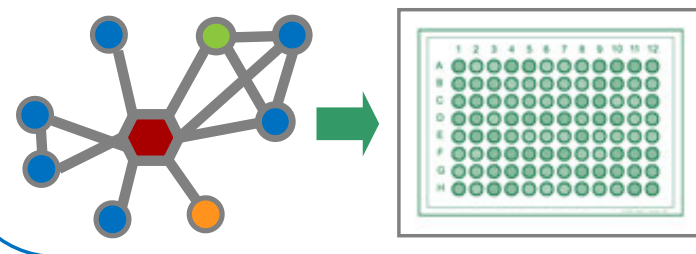

Gene knockdowns scored by similarity to BAG3 knockdown

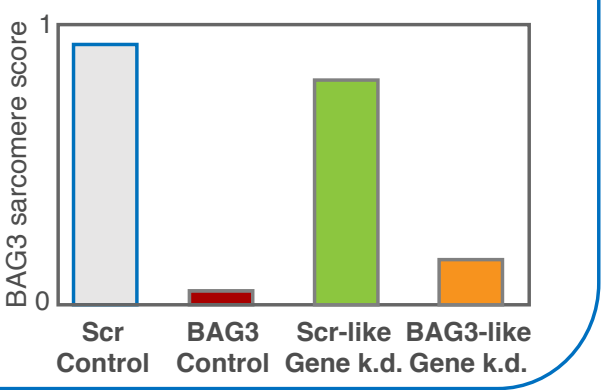

B
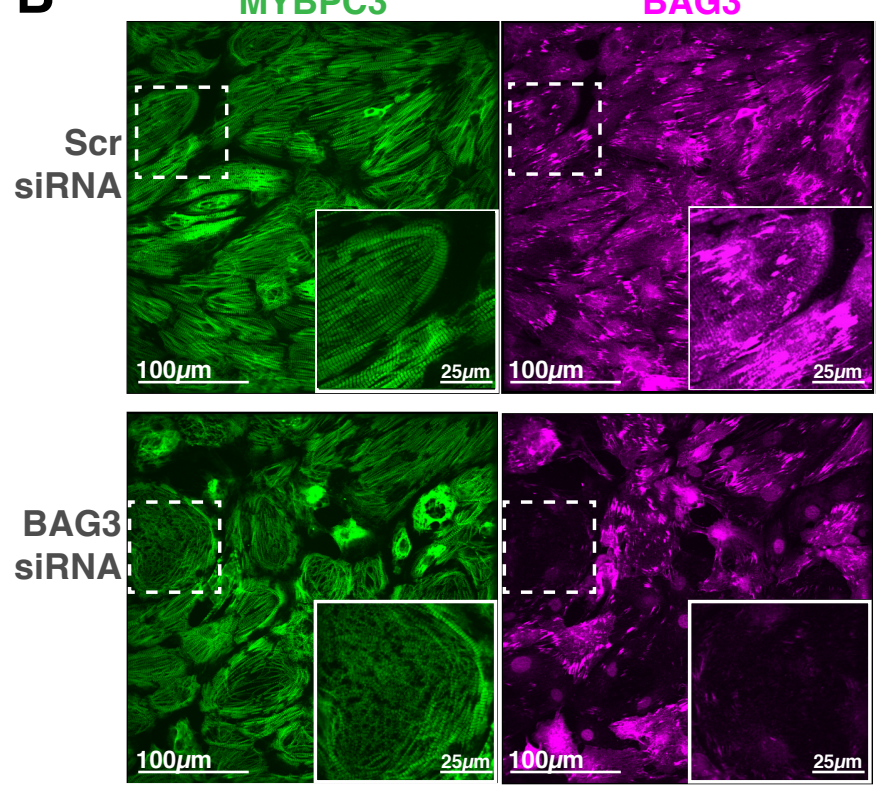

MYBPC3 BAG3 DAPI

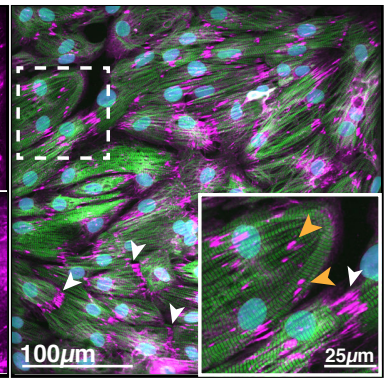

D

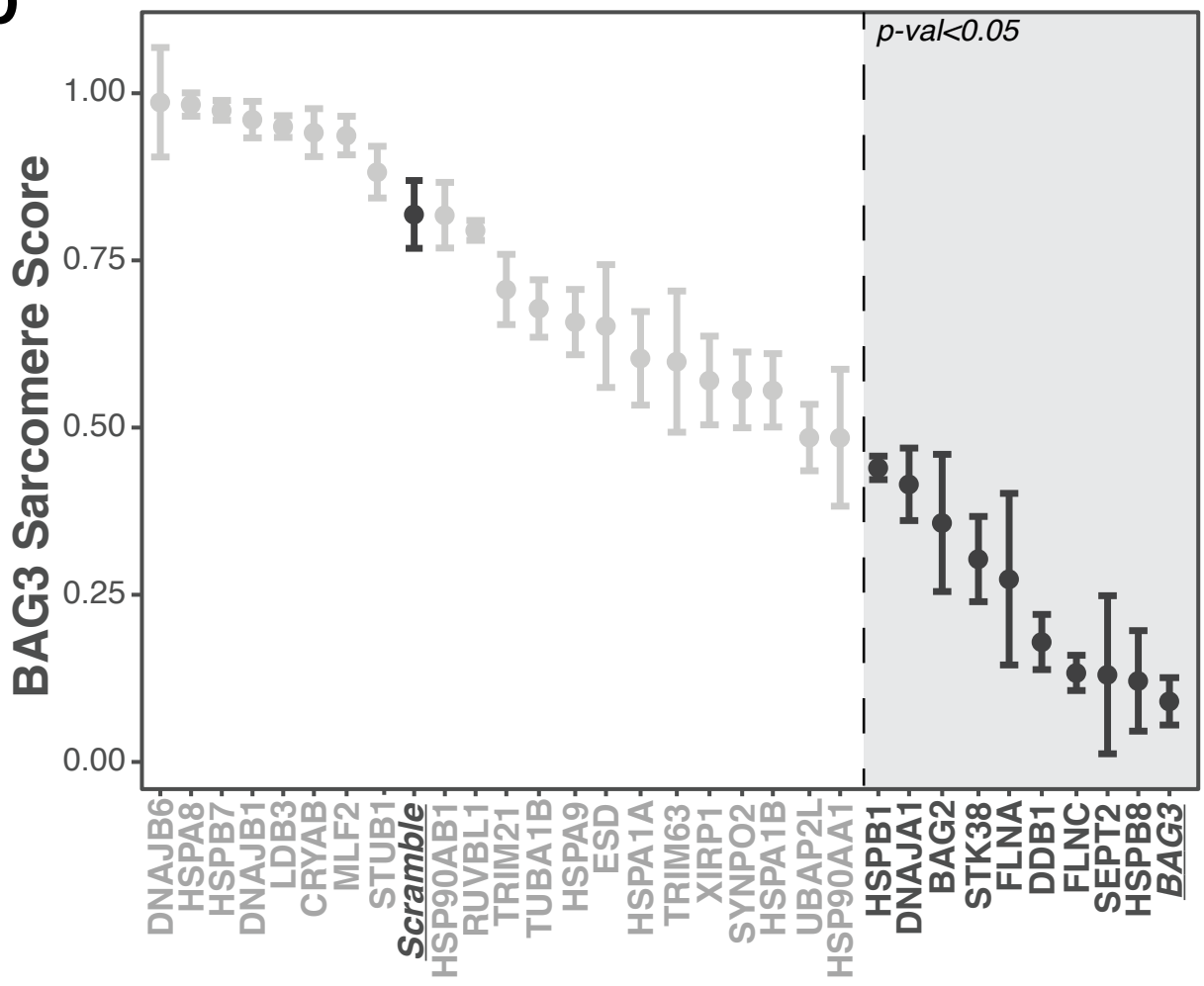

C

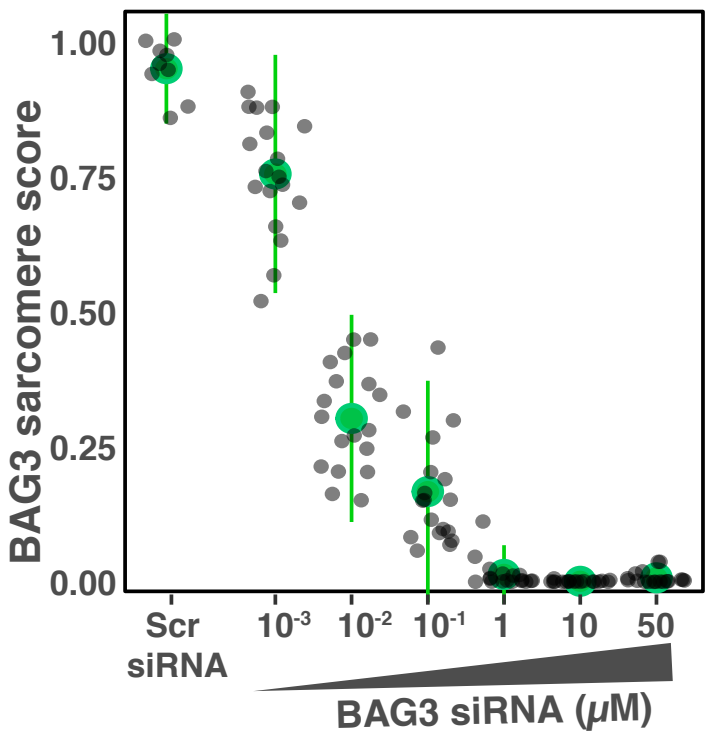

E

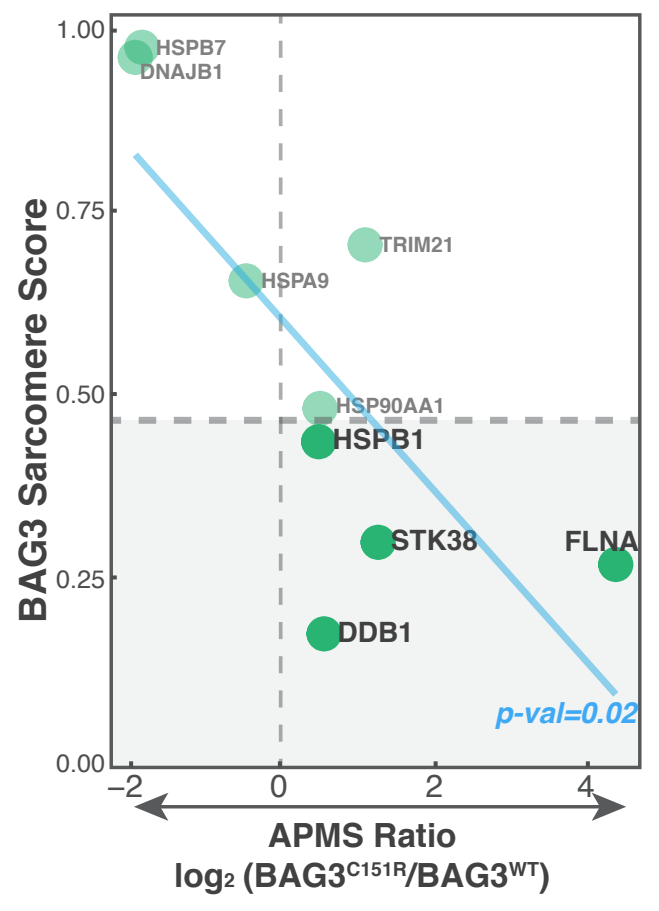




\section{Figure 2. BAG3 $3^{\mathrm{C} 151 \mathrm{R}}$ favors binding to factors required for maintenance of myofibrillar} integrity. (A) Diagram of the pipeline followed for automated analysis of myofibrillar staining images in iPS-CM. First, BAG3 associated genes are knocked down using siRNA. Then those images are put through a supervised learning scoring scheme trained on myofibrillar staining images of iPS-CM treated with Scramble (control) or BAG3 siRNA. Scores are closer to 1 if the image resembles a scramble condition and closer to 0 if similar to a BAG3 knockdown, as shown in the mock graph in A. (B) Representative images of iPS-CMs treated with control or BAG3 siRNA. BAG3 staining localized to the sarcomeric z-disk region and with high intensity in the MYBPC3 staining gaps (presumably myofibrillar breaks; orange arrowheads) and, in some cases, to the polar ends of cardiomyocytes (white arrowheads). BAG3 knockdown cardiomyocytes display a reduced density of myofibrils, which are also more disorganized, and more cells with collapsed/aggregated sarcomere staining (yellow arrowheads). Cells with the lowest BAG3 staining displayed a particularly disorganized sarcomere structure, with numerous gaps in MYBPC3 staining (see insert). (C) Image score correlate anticorrelated with the amount of BAG3 siRNA used. $N=9$ images for Scr siRNA condition, $N=18$ for the rest. (D) BAG3 sarcomere score for all the BAG3 co-precipitation partners identified in this study. Internal controls Scramble and BAG3 are underlined. Vertical dashed line marks the threshold beyond which a knockdown scored significantly lower than the internal Scr control (no condition scored significantly higher than Scramble). Dots represent mean of 3 replicates from separate wells, each being the median score of 9 images from the same well. Error bars: SEM. P-val cutoff: 0.05 using a one-way ANOVA with post-hoc Dunnett test. (E) Knocking down proteins that interact stronger with BAG3 ${ }^{\mathrm{C} 151 \mathrm{R}}$ than $\mathrm{BAG} 3^{\mathrm{WT}}$ phenocopies BAG3 insufficiency. Plot depicting the relationship between the sarcomeric score of a gene knockdown and the corresponding protein's BAG3 ${ }^{\mathrm{C} 151 \mathrm{R}} / \mathrm{BAG}{ }^{\mathrm{WT}}$ co-precipitation intensity ratio. $\mathrm{P}$-value obtained fitting a linear model. Pearson's product-moment correlation: -0.75 . 
181 FLNA, STK38, DDB1 and HSPB1, proteins that more strongly co-precipitated with 182 BAG3 ${ }^{C 151 R-3 \times F L A G}$ than BAG3 ${ }^{W T}$, were among the phenotypes that most resembled

183 BAG3 insufficiency. In fact, we observed an inverse relationship between the BAG3

184 sarcomere scores and the change in co-precipitation intensity for all BAG3 ${ }^{C 151 R-3 \times F L A G}$

185 differential interactors (Fig 2E). This correlation was not observed for BAG3 ${ }^{\mathrm{E} 455 \mathrm{~K}}$

186 differential binding partners (Fig S7G).

Altogether, this data implicates a specific subset of BAG3 interactors as particularly important for the maintenance of myofibrillar integrity. It also suggests that BAG3 ${ }^{C 151 R}$ variant has an increased ability to engage some of these specific factors,

191 presumably improving their BAG3-associated role in the maintenance of the sarcomeric 192 structure.

\section{BAG3-C151R protects against toxic proteasome inhibition in cardiomyocytes}

Since BAG3 is a well-known stress responsive factor with a role in the maintenance of cell homeostasis under proteotoxic conditions, we next wanted to elucidate whether the BAG3 ${ }^{\mathrm{C} 151 \mathrm{R}}$ variant had a measurable effect in cardiomyocyte response to stress. To this end, we generated two additional isogenic iPSC lines bearing the rs2234962/BAG3 ${ }^{\text {C151R }}$ variant in either heterozygosity or homozygosity. This way we would be able to study gene-dose effects. We exposed iPS-CMs from these cell lines to increasing doses of the proteasomal inhibitor bortezomib, a well-known cardiotoxic drug ${ }^{36-38}$ to which BAG3-deficient cells are particularly sensitive 25,34 .

204 Interestingly, iPS-CMs bearing BAG3 ${ }^{\mathrm{C} 151 \mathrm{R}}$ variants showed a higher $\mathrm{EC}_{50}$ than $\mathrm{BAG} 3^{\mathrm{WT}}$ 205 cardiomyocytes did, indicating a greater resilience to the inhibitor. This effect was 206 stronger in BAG3 ${ }^{\mathrm{C} 151 \mathrm{R}}$ homozygous cardiomyocytes than in heterozygous ones, 207 suggesting a response influenced by gene dose (Fig 3A, Fig S7A). By contrast, , 208 cardiomyocytes expressing the pathogenic BAG3 ${ }^{\mathrm{E} 455 \mathrm{~K}-3 x F L A G}$ variant were significantly 209 more susceptible to bortezomib than BAG3 ${ }^{W T}$ cardiomyocytes (Fig 3A, Fig S9A), and 210 had an $\mathrm{EC}_{50}$ similar to that of $\mathrm{BAG}^{-/-}$cell lines, consistent with observations that 211 pathological BAG3 mutations that impact the BAG domain can have a dominant 
212 negative effect ${ }^{29}$. We also noted that BAG3 overexpression was able to rescue the 213 BAG3 $^{-/-}$phenotype and provide an additional level of resistance over BAG3WT (Fig

214 S7A-B), in agreement with studies using BAG3 overexpression as a potential

215 cardioprotective strategy 39,40 .

216

Finally, to rule out that the change in cardiomyocyte response to stress was due

218 to an increase in BAG3 protein levels, we quantified protein expression in iPS-CM from

219 the cell lines bearing $B A G 3^{C 151 R}$ and $B A G 3^{E 455 K}$ variants; we found no significant

220 changes in protein levels, compared to BAG3 ${ }^{\mathrm{WT}}$ (Fig S9C).

221

222 These results show that the $B A G 3^{C 151 R}$ variant provides an enhanced, dose-

223 dependent resistance to proteotoxic stress in cardiac muscle cells in vitro, and that a 224 single amino acid change rather than a change in protein levels is responsible for this 225 effect. A proposed mechanism for the influence of single nucleotide variants on BAG3 226 interactions and iPS-CM response to stress is show in (Fig 3B). 


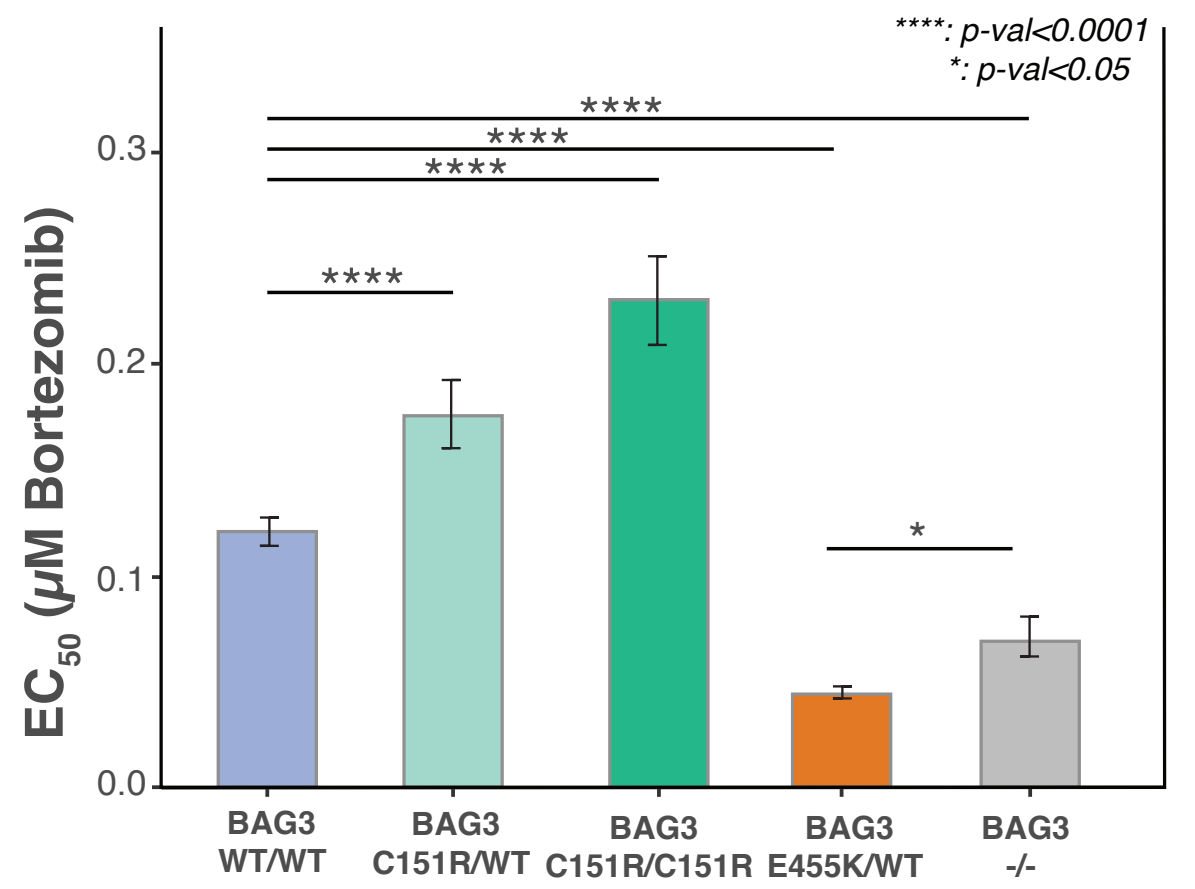

B

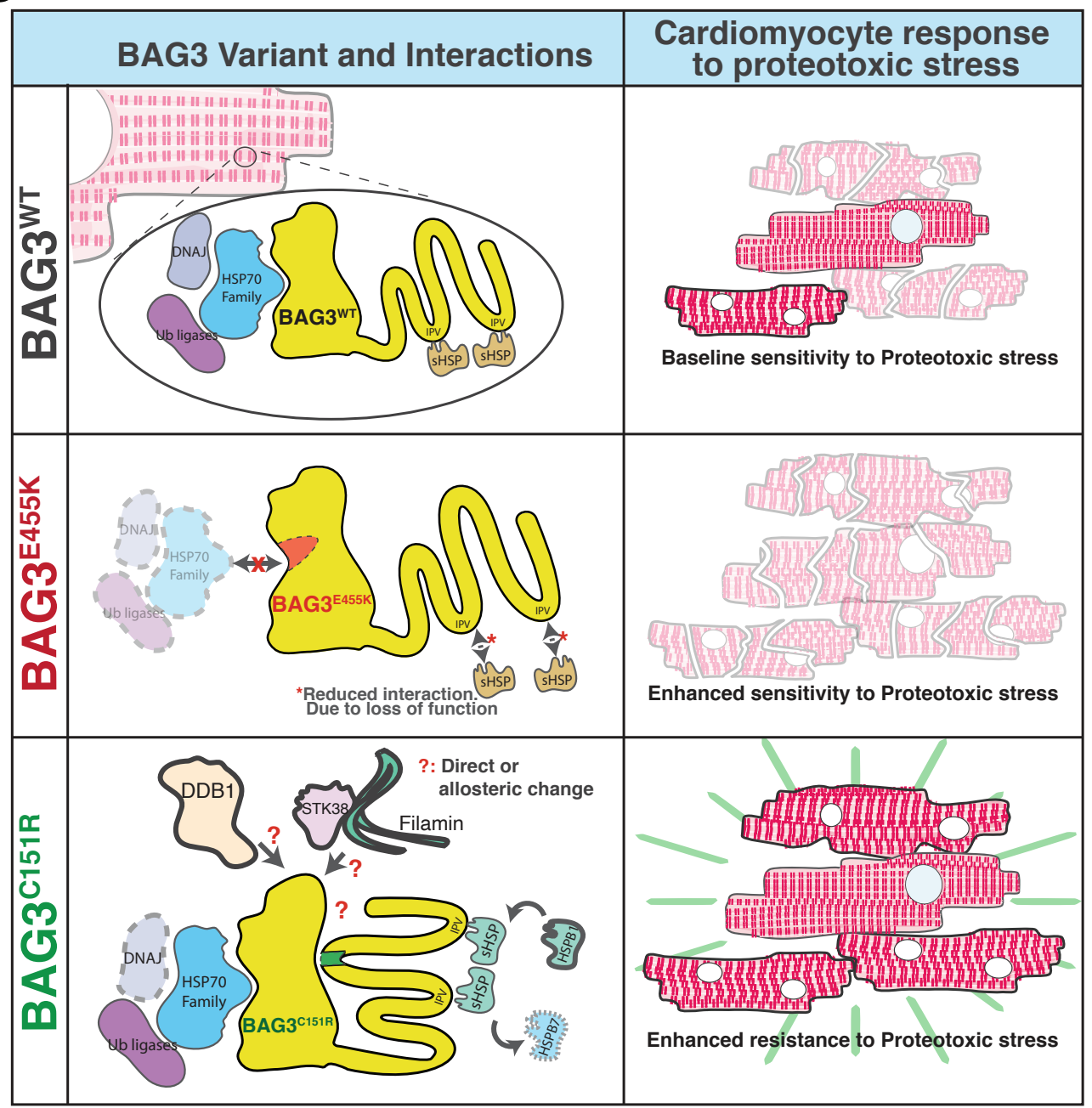

Figure 3. The rs2234962BAG3 ${ }^{\mathrm{C} 151 \mathrm{R}}$ variant provides enhanced resistance to the proteotoxic drug bortezomib in iPS-CM. (A) Calculated EC 50 and $95 \%$ confidence intervals for Bortezomib in control (WT/ WT), BAG3 ${ }^{\mathrm{C} 151 \mathrm{R}}$ heterozygous (C151R/WT) and BAG3 ${ }^{\mathrm{C} 151 \mathrm{R}}$ homozygous (C151R/C151R) iPS-CMs. Cells bearing the pathogenic variant $B A G 3^{\mathrm{E} 455 \mathrm{~K}}$ (along with a $3 x F L A G$ epitope) and cells lacking BAG3 expression were used as a positive control for increased sensitivity to bortezomib. $N=3$. ****: P-value $<0.0001$ and *: $p$ value $<0.5$ using one-way ANOVA with post-hoc Zidak correction. (B) Graphic summary of the data presented in this study: BAG3 ${ }^{\mathrm{C} 151 \mathrm{R}}$ changes the profile of BAG3 protein interactions (mediated by a hypothetical structure conformational change or stabilization of a particular structural state or transition) toward factors required for cardiomyocyte myofibrillar quality control (DDB1, STK38, Filamin). This in turn results in an enhanced resistance to proteotoxic insult. It is unclear if the increased binding of myofibril quality control factors is driven by a direct interaction with the local amino acid sequence or by an allosteric effect. 


\section{Discussion}

In this study, we report that the common variant rs2234962-which results in the $B A G 3^{C 151 R}$ amino acid change-causes a change in the interaction profile of the BAG3

230 protein and is directly associated with a heightened resistance of human

231 cardiomyocytes to proteotoxic stress. Although numerous studies have associated the

232 haplotype block containing this variant to a lower incidence of DCM, direct causality had

233 been hard to infer from genetic association studies alone, in part due to the multiple

234 linked nucleotide variants in this region, and the lack of physiological relevant models to 235 study such cardioprotective effects. Since increasing BAG3 protein levels has been 236 shown to have a cardioprotective effect ${ }^{39,40}$, we considered two hypotheses: 1) that the

237 C151R amino acid change directly modifies BAG3 function in a manner leading to 238 cardioprotective effects and 2) that the haplotype block functions primary by regulating 239 BAG3 expression. Here, we sought to test the first hypothesis. We utilized genome 240 engineering to precisely insert the $B A G 3^{C 151 R}$ variant in an otherwise unchanged genetic 241 background, and used disease modeling powered by iPSCs to identify a protective 242 effect on cardiomyocytes exposed to a cardiotoxic drug. Although we have not tested 243 the effect of other genetic variants in the same haplotype block, our observation that 244 BAG3 ${ }^{C 151 R}$ is sufficient for a functional change without a significant change in protein

245 levels strongly suggests that this coding change is driving at least some of the 246 cardioprotective effect observed in genetic association studies. Our data also indicates 247 an allele dosage effect for this protection, in agreement with some genetic studies that 248 suggest homozygous individuals have an even lesser risk of disease than heterozygous 249 individuals ${ }^{5,13}$.

This study demonstrates the strength genetically engineered iPSCs to dissect the 252 effects of human genetic variants on cardiac myopathies. The ability to perform genome 253 engineering techniques on iPSCs and the increasing breadth of functional information 254 that can be obtained from iPS-CMs enables prospective studies in a cost- and time255 efficient manner. Here, in addition to characterizing cardiomyocyte-specific protein 256 interactions, we used an automated machine learning pipeline to objectively analyze 257 microscopy images from different BAG3-associated gene knockdowns. This adds a 
layer of information to the putative interaction list by quantifying the contribution of each one of the factors to the myofibrillar structure, allowing us to prioritize targets by identifying which ones are involved in BAG3-associated maintenance of sarcomeric integrity. A similar approach has been used to study small molecule perturbations on

262 iPS-CMs and other cell types ${ }^{41,42}$. The use of iPS-CMs as a model for cardiomyopathies is not without limitations: iPS-CMs are known to lack some important features of mature

264 myocytes and are not exposed to mechanical stress from pressure load, which is a

265 predominant factor in the challenging of the myocardial protein quality control

266 machinery $28,43-45$. This last point could influence the profile of BAG3 interactions and

267 might explain the lower representation of structural and sarcomeric proteins in our

268 BAG3 interactome compared to other similar studies using primary heart tissue ${ }^{27,28}$.

269 Despite the highlighted differences between iPS-CMs and mature cardiomyocytes, we

270 and others have been able to identify functional changes in genome-edited iPS-CMs

271 that are consistent with the observations from human genetic association

272 studies $25,34,44,45$. Here, we used the proteasome inhibitor bortezomib as an inducer of

273 proteotoxic stress. Bortezomib is a chemotherapy drug with a marked cardiotoxic effect,

274 which acts by stalling the proteasome networks and increasing the accumulation of

275 misfolded proteins ${ }^{46}$. This buildup of toxic protein species is also a hallmark of the aging

276 and heart failure myocardium, presenting bortezomib insult as a useful tool to study

277 cardiac protein quality control. This correlation between in vitro results and genetic

278 studies suggests that the role of BAG3 in iPS-CM is at least partially representative of

279 its function in the adult human heart muscle. In addition, we were only able to identify

280 significant changes in co-precipitation with the BAG3 ${ }^{\mathrm{C} 151 \mathrm{R}}$ when using a cardiomyocyte

281 background, stressing the importance of using a disease-relevant cell model for the

282 functional study of cardiomyopathy disease variants.

The differences in co-precipitation partners between the $\mathrm{BAG} 3^{\mathrm{C} 151 \mathrm{R}}$ and the wild type protein point towards a molecular mechanism for the cardioprotection observed in 286 genetic association studies. The largest increase in binding was observed for Filamin A 287 (FLNA). The gene for FLNA is expressed at high level in embryonic cardiomyocytes, but 288 its expression goes down in the adult striated muscle in favor of a paralog, Filamin 
$289 C^{47,48}$. Although we also identified FLNC in our pulldown samples, the high abundance

290 of Filamin A in iPS-CM could be facilitating an interaction in this cellular background.

291 The importance of FLNA in iPS-CM is also highlighted by the strong phenotype

292 observed in our gene knockdown analyses. The BAG3 ${ }^{\text {C151R }}$ variant also showed a

293 significant ( 2-fold) enrichment on the hippo pathway kinase STK38/NDR1, which was

294 recently described as an inhibitor of BAG3-triggered autophagy ${ }^{49}$, and ubiquitin ligases

295 DDB1 and TRIM21, which could potentially aid in the BAG3-mediated disposal of

296 ubiquitinated defective proteins ${ }^{26}$. Both HSPB1 and STK38/STK38L have been

297 independently described to mediate Filamin phosphorylation ${ }^{50,51}$. We also observe a

298 reduction in the co-precipitation of cardiac-specific small heat shock protein HSPB7.

299 Although this protein has been previously described to not interact directly with BAG3 ${ }^{52}$,

300 we identify it in our co-precipitation purification studies, suggesting it may be an indirect

301 interaction through other interaction partners (for example, HSPB8 ${ }^{53}$ ) and that the

302 BAG3 ${ }^{\text {C151R }}$ change may tilt the sHSP composition of BAG3 complexes in favor of other

303 members (such as HSPB1). Residue 151 is located in a conserved region of the BAG3

304 protein with no known domain or function (see Figure S1A). Due to its proximity to the

305 IPV domains, which mediate interaction with $\mathrm{SHSPS}^{54}$, the BAG3 ${ }^{\mathrm{C} 151 \mathrm{R}}$ transition could

306 be directly affecting the binding of these. Alternatively, due to the predicted intrinsically

307 disordered nature of this segment of BAG3, it is possible that such change could have

308 an effect on distant regions of the protein, such as the BAG domain that is responsible

309 for indirectly binding of BAG3 protein targets for quality control.

310

It is particularly interesting that knockdown of the BAG3 ${ }^{\mathrm{C} 151 \mathrm{R}}$ differential

312 interactors scored as being closely related to BAG3 insufficiency in our myofibrillar

313 staining image analyses. BAG3 is a known protein hub that dynamically connects

314 different subcellular processes resulting in a broad functional spectrum ${ }^{21,54}$. We propose

315 that the $\mathrm{BAG} 3^{\mathrm{C} 151 \mathrm{R}}$ protein variant tilts the balance of interactions to favor a number of

316 factors that are required for the maintenance of myofibrillar structure in a BAG3

317 dependent manner (FLNA, DDB1, STK38, HSPB1 among others) (Fig 3B). This could in

318 turn lead to enhanced function by either interaction stabilization or more effective 
319 detection and disposal of misfolded species. Future studies will be required to test these

320 and other possible models for the BAG3 ${ }^{\mathrm{C} 151 \mathrm{R}}$ mechanism of action.

321

322

To our knowledge, the data presented here provides the first evidence of a

323 functional change associated with the rs2234962/BAG3 ${ }^{\mathrm{C} 151 \mathrm{R}}$ variant, and the first time a

324 putatively protective hit from a heart failure genetic association study has been validated

325 in vitro. In the human heart, cardiomyocytes are under constant physical stress and

326 have very limited proliferative potential, making them rely on an active proteostasis

327 network to properly assemble and dispose of myofibrillar factors. The BAG3 co-

328 chaperone is a key member of this machinery, and loss of protein expression or loss-of-

329 function variants are known to lead to dilated cardiomyopathy and heart failure. Our

330 study shows that a specific amino acid change in the BAG3 protein can also lead to the

331 opposite effect, cardioprotection. Despite the high frequency of BAG3 ${ }^{\mathrm{C} 151 \mathrm{R}}(\sim 20 \%$ in

332 European and South-East Asian populations), no negative health effects have been

333 described to date for this variant. This opens an avenue for further exploration of

334 therapeutic strategies that tackle the role of BAG3 in the heart, particularly those aiming

335 to mimic the effect of the $B A G 3^{C 151 R}$ variant, as a way to improve cardiac muscle

336 function and aid in the treatment or prevention of hereditary and acquired heart failure. 


\section{Methods}

\section{BAG3 genetics and conservation plots}

Linkage disequilibrium plots for the BAG3 putative protective allele block were generated using data downloaded from the International Genome Sample Resource (IGSR) database ${ }^{55}$, using the data for the CEU population (Utah residents with Northern

343 and Western European ancestry). The map graphs with the geographic distribution of

344 allele frequencies for the rs2234926 variant was obtained using the Geography of

345 Genetic Variants (GGV) browser tool ${ }^{56}$ and edited in-house to enhance readability. Both

346 tools used population data from the 1000 Genomes Consortium ${ }^{57}$. Aminoacid

347 conservation data and zoomed-in plots were obtained from Aminode database ${ }^{58}$.

iPSC culture and differentiation into iPS-CM

The WTC iPSC line was derived from a healthy male volunteer and is widely

351 used as a normal control. WTC and derived cell lines were maintained and

352 differentiated as previously described ${ }^{25}$. Briefly, for routine maintenance, cells were

353 cultured on Growth Factor Reduced Matrigel ( $8 \mu \mathrm{g} / \mathrm{ml}$, BD Biosciences) and maintained

354 in mTesr1 medium (STEMCELL Technologies) every day. Whenever the cells reached

$35570-90 \%$ confluence, they were harvested using Accutase Cell Detachment Solution

356 (STEMCELL Technologies) or ReLeSR Human Pluripotent Stem Cell Passaging

357 Reagent (STEMCELL Technologies), and maintained on media supplemented with the

358 ROCK1 inhibitor Y-27632 (10 $\mu \mathrm{M}$, Selleckchem) for the first 24 hours. Cardiomyocyte

359 differentiation from iPSCs was performed as previously described ${ }^{25}$. Briefly, iPSCs were

360 seeded on Matrigel-coated plates, at a density between $6.25 \times 10^{3}$ and $2.5 \times 10^{4}$

361 cells $/ \mathrm{cm}^{2}$. When cells reached $40-80 \%$ confluence (day 0 ), media was changed to

362 RPMI1640 (Gibco) with B27 supplement (without insulin; ThermoFisher)) containing 6

$363 \mu \mathrm{M}$ or $12 \mu \mathrm{M}$ CHIR99021 (Tocris) for 24 or 48 hours. Initial seeding, confluence level at

364 day 0 and optimal concentration and exposure time for CHIR were optimized for each

365 line. Cells were then incubated in RPMI/B27 (-Insulin) for 24 hours before changing

366 media to $5 \mu \mathrm{M}$ IWP2 (Tocris) in RPMI/B27(-Insulin). After another $48 \mathrm{~h}$, media was 
changed to RPMI/B27 containing insulin. Fresh RPMI/B27 was exchanged every 3-4 days thereafter, and cells were monitored for beating daily.

\section{iPS-CM enrichment and cryopreservation}

At day 15 , differentiation cultures that showed beating in $>20 \%$ of surface area were harvested using $0.25 \%$ Trypsin (Gibco) and replated. For enrichment of iPS-CMs, we used a metabolic selection protocol as previously described ${ }^{25}$. Briefly, cells were

374 allowed to recover for 72 hours, and then media was thoroughly washed with PBS and

375 replaced with DMEM (no glucose, with sodium pyruvate, ThermoFisher Scientific)

376 supplemented with Glutamax, Non-Essential Amino Acids, and buffered lactate (4 mM).

377 Cells were treated with lactate media for 48 hours, three consecutive times. Then cells

378 were allowed to recover in RPMI/B27(+Insulin). For each differentiation batch, a

379 separate well was prepared in parallel to be used for cell counting, quantification of

380 differentiation efficiency and genotyping. Differentiation efficiency was quantified using

381 flow cytometry as described previously ${ }^{59}$ using mouse monoclonal antibody against

382 cardiac troponin-T (clone 13-11, Thermo Fisher Scientific). All iPS-CM cultures used in

383 this study were $>85 \%$ cTnT+, day 30 . For cryopreservation, day-30 enriched iPS-CM

384 cultures were harvested using $0.25 \%$ Trypsin and stored in CryoStor Cell Freezing

385 Medium (STEMCELL Technologies) in liquid nitrogen. For thawing, cells were plated in

386 thawing media (RPMI/B27(+Insulin) supplemented with $10 \mu \mathrm{M} \mathrm{ROCK}$ inhibitor and $20 \%$

387 HyClone Fetal Bovine Serum (Fisher Scientific) and directly seeded onto cell culture

388 plates as desired. Two hours later, media was replaced and cells were maintained as

389 described above.

390

\section{Genome engineering of iPSC lines}

392 Derivation of the BAG3 ${ }^{-1-}, B A G 3^{\text {WT-FLAG }}$ and BAG3 ${ }^{\mathrm{C} 151 \mathrm{R}}$ (no $3 \times$ XLAG fusion) from

393 the WTC cell line has been described previously ${ }^{25,60}$. For the generation of the

$394 \mathrm{BAG}^{\mathrm{E} 455 \mathrm{~K}}$ cell line, we introduced the corresponding single nucleotide mutation in the

395 3xFLAG donor plasmid using QuikChange Site-Directed Mutagenesis Kit (Agilent).

396 Then this donor plasmid was used for the same process used to generate the BAG3 ${ }^{W T-}$ 
397 FLAG line ${ }^{25}$. To generate the inducible expression cell line BAG3 ${ }^{-/-}$: TetON-BAG3WT-3xFLAG, 398 we used a TALEN pair described elsewhere ${ }^{61}$. Two million WTC iPSC were 399 nucleofected using an Amaxa nucleofector 2B and the Nucleofector Kit C (both from 400 Lonza), $0.5 \mu \mathrm{g}$ of each AAVS1 TALEN pair and $1 \mu \mathrm{g}$ of the donor plasmid (see Figure 401 S4). Cells were then selected on $1 \mathrm{mg} / \mathrm{ml} \mathrm{G418,} \mathrm{before} \mathrm{picking} \mathrm{single} \mathrm{clonal} \mathrm{populations}$ 402 for genotyping. To generate the BAG3 ${ }^{C 151 R / W T}$ and BAG3 ${ }^{C 151 R / C 151 R}$ knock-in cell lines, $4038 \times 10^{5}$ WTC iPSC were nucleofected using Amaxa nucleofector 2B and Solution P3 404 (Lonza), 80 pmol Truecut v2 Cas9 (Life Technologies), 24 0pmol sgRNA (Synthego; 405 ACACUGUUUAUCUGGCUGAG), and 400 pmol single-stranded donor (IDT; 406 ctcagaggtcccagtcacctctgcggggcatgccagaaacaactcagccagataaacagcgtggacaggtggctgca 407 gcggcggcagcccagccccagcctcccacggacctgagg). A list of all the cell lines generated and 408 used in this study can be found in Table MM.1.

Table MM.1 - Cell lines generated in this study. *: HGVS nomenclature as per guidelines of the Human Genome Variation Society ${ }^{62}$. **: Short name used throughout 412 this manuscript, based on protein level changes.

\begin{tabular}{|l|l|l|}
\hline HGVS name* & Short name** $^{*}$ & Notes \\
\hline BAG3 & BAG375T-3xFLAG & $\begin{array}{l}\text { C-terminal fusion of the 3xFLAG } \\
\text { epitope sequence. }\end{array}$ \\
\hline $\begin{array}{l}\text { BAG3 } \\
\text { p.[C151R; } \\
\text { P575_576ins3xFLAG];[=] }\end{array}$ & $\begin{array}{l}\text { BAG3 }{ }^{\text {C151R- }} \\
\text { 3xFLAG/wt }\end{array}$ & $\begin{array}{l}\text { The amino acid transition and the } \\
\text { 3xFLAG fusion are in the same } \\
\text { allele (in cis) }\end{array}$ \\
\hline $\begin{array}{l}\text { BAG3 } \\
\text { p.[E455K; } \\
\text { P575_576ins3xFLAG];[=] }\end{array}$ & $\begin{array}{l}\text { BAG3 }{ }^{\text {E455K- }} \\
\text { 3xFLAG/wt }\end{array}$ & $\begin{array}{l}\text { The amino acid transition and the } \\
\text { 3xFLAG fusion are in the same } \\
\text { allele (in cis) }\end{array}$ \\
\hline
\end{tabular}




\begin{tabular}{|l|l|l|}
\hline $\begin{array}{l}\text { PPP1R12C } \\
\text { p. }[=][=][\text { TetOn- } \\
\text { BAG3*ins3xFLAG] }\end{array}$ & $\begin{array}{l}\text { BAG3--: TetON- }^{*} \text { BAG3 } \\
\text { BT-3xFLAG }\end{array}$ & $\begin{array}{l}\text { Insertion of a cassette expressing } \\
\text { the cDNA of BAG3 in the } \\
\text { PPP1R12C (AAVS1) locus }\end{array}$ \\
\hline BAG3 p.C151R & BAG3 $^{\text {C151R/WT }}$ & $\begin{array}{l}\text { Heterozygous endogenous } \\
\text { knock-in }\end{array}$ \\
\hline BAG3 p.C151R & BAG3 ${ }^{\text {C151R/C151R }}$ & $\begin{array}{l}\text { Homozygous endogenous knock- } \\
\text { in }\end{array}$ \\
\hline
\end{tabular}

\section{Cell line genotyping, ddPCR linkage assay and karyotyping}

417 For sequencing validation of genotypes, target genomic regions were amplified 418 by PCR with BioMix Red (Bioline), sent for Sanger sequencing by MCLAB (South San 419 Francisco, California) or Quintara Biosciences (South San Francisco, California).

420 Quantification of the allelic abundance of the modifications inserted and verification of 421 the excision of selection cassettes was performed by droplet digital PCR (ddPCR). For 422 each ddPCR reaction, 50 or 100ng of genomic DNA was mixed with $5 \mu \mathrm{M}$ of each one 423 of two TaqMan MGB detection probe (FAM or HEX/VIC), $18 \mu \mathrm{M}$ of forward and reverse 424 amplification primer, and 1x of ddPCR Supermix for Probes (Bio-Rad). This mix was 425 processed into droplets using a QX100 Droplet Generator (Bio-Rad) and the emulsion 426 was transferred into a 96-well plate for thermal cycling. Amplifications were performed 427 on a C1000 Thermal Cycler (Bio-Rad), using the following settings: step $1,95^{\circ} \mathrm{C}$ for 10 428 minutes; step $2,94^{\circ} \mathrm{C}$ for 30 seconds; step 3, annealing temperature (optimized for 429 each probe pair) for 30 seconds; repeat steps 2-3 39 times; then step 4, $98^{\circ} \mathrm{C} 10$ 430 minutes. Fluorescence readouts for each droplet were obtained using a QX100 Droplet 431 Reader (Bio-Rad) in the "absolute quantification" setting. To estimate the abundance of 432 single nucleotide variant alleles, probe pairs were designed to discriminate between 433 original and edited sequence. A ratio of modified vs original positive droplets was used 434 as a readout (i.e. $\sim 1: 1$ for heterozygous). For copy number variation analysis of the 
$4353 x F L A G$ and puromycin cassettes, the ratio of droplets positive for these sequences

436 versus droplets positive to a reference gene (RPP30 PrimePCR ${ }^{\text {TM }}$ Probe Assay,

437 BioRad) was used as a readout. Primers and probes for ddPCR assays were designed

438 using the TaqMan MGB Allelic Discrimination option in Primer Express 3.0 software

439 (Life Technologies). Sequences of the primers used in the genotyping reactions are

440 described in Table MM.2.

For allelic phasing of the BAG3 variants and the 3xFLAG sequence, a ddPCR443 based assay was used (See Fig S2). This assay combined a TaqMan probe detecting

444 the 3xFLAG cassette and a probe binding the single-nucleotide variant we wished to 445 phase. Droplet digital PCR was performed as described above, and the linkage 446 percentage for variants was computed as described elsewhere ${ }^{63}$.Briefly, assuming

447 independence between events, then: $\frac{\left(N_{A} * N_{B}\right)}{N_{A B}}=1-\left(N_{A}+N_{B}+N_{A B}\right)=N_{\text {empty }}$;

$448\left(N_{E} * N_{A B}\right)=N_{A} * N_{B}$, where $\mathrm{N}_{\mathrm{A}}$ and $\mathrm{N}_{\mathrm{B}}$ are the number of droplets positive for event $\mathrm{A}$ 449 and $B$, respectively, while $N_{A B}$ are double-positive droplets due to chance and $N_{E}$ are 450 empty droplets. If the case where the two events are physically linked is $\mathrm{N}_{\underline{A B}}$, and using 451 the Poisson statistics equation for where where $\lambda_{x}=-\ln \left(\lambda_{\text {not } x}\right)$, we obtain: $\lambda_{\overline{A B}}=$ $452-\ln \left(\frac{N_{A}+N_{B}+N_{E}+\frac{N_{A} N_{B}}{N_{E}}}{N_{\text {all }}}\right) ; \lambda_{A}=-\ln \left(\frac{N_{\text {not } A}}{N_{\text {all }}}\right) ;$ and $\lambda_{B}=-\ln \left(\frac{N_{\text {not } B}}{N_{\text {all }}}\right)$. The estimated \% of 453 linked molecules is then calculated as: $\% \overline{A B}=\frac{\lambda_{\overline{A B}}}{\frac{\left(\lambda_{A}+\lambda_{B}\right)}{2}} * 100$.

Karyotyping of the cells was performed by Cell Line Genetics LLC (Madison,

456 Wisconsin). Only cell lines with apparently normal karyotype were used in this study.

458 Table MM.2 - Sequences of primers and probes for genotyping reactions

\begin{tabular}{|l|l|}
\hline Assay Description & Sequences \\
\hline BAG3 ${ }^{3 x}$ FLAG upstream arm junction & F: TGCCATTAAGAATACCATCTACAGAG \\
sequencing & R: GTGGTCCTTGTAGTCGCC \\
\hline
\end{tabular}




\begin{tabular}{|c|c|}
\hline $\begin{array}{l}\text { BAG3 }{ }^{3 x F L A G} \text { downstream arm junction } \\
\text { sequencing }\end{array}$ & $\begin{array}{l}\text { F: CTACAAGGACGACGACGA } \\
\text { R: CACCACCTGGCTGATTTG }\end{array}$ \\
\hline BAG3 ${ }^{3 x F L A G} 3 x F L A G$ sequencing & $\begin{array}{l}\text { F: GTCTATGAACTCCAGCCCAG } \\
\text { R: CTACAAAAGACAGTGCACAAC }\end{array}$ \\
\hline 3xFLAG (ddPCR) & $\begin{array}{l}\text { F: GGTCAAGTCCAGGTCTATGAAC } \\
\text { R: TTGTCGTCGTCGTCCTT } \\
\text { Probe:ATCCGACTACAAGGAC }\end{array}$ \\
\hline $\begin{array}{l}\text { Puromycin resistance copy number variation } \\
\text { (ddPCR) }\end{array}$ & $\begin{array}{l}\text { F: GTCACCGAGCTGCAAGAA } \\
\text { R: CACCTTGCCGATGTCGAG } \\
\text { Probe:CTCTTCCTCACGCGCGTCGG }\end{array}$ \\
\hline $\begin{array}{l}\text { Neomycin resistance copy number variation } \\
\text { (ddPCR) }\end{array}$ & $\begin{array}{l}\text { F: CATGGCTGATGCAATGCG } \\
\text { R: TCGCTTGGTGGTCGAATG } \\
\text { Probe: CGCTTGATCCGGCTACCTGCC }\end{array}$ \\
\hline BAG3 ${ }^{\mathrm{C} 151 \mathrm{R}} \mathrm{ddPCR}$ & $\begin{array}{l}\text { F: CAGCAGCGGCTCCTCAGA } \\
\text { R: GTGAGCCTGGCCTCTCCTT } \\
\text { Probe (WT): CAGATAAACAGTGTGGACA } \\
\text { Probe (C151R) AGATAAACAGCGTGGACA }\end{array}$ \\
\hline$B A G 3^{\mathrm{E} 455 \mathrm{~K}} \mathrm{ddPCR}$ & $\begin{array}{l}\text { F: TGAAGGCAAGAAGACTGACAAAAA } \\
\text { R: TCCTGGCCTGACGCACAT } \\
\text { Probe (E455K): TACCTGATGATCAAAG }\end{array}$ \\
\hline WT AAV F & $\begin{array}{l}\text { F: CGGTTAATGTGGCTCTGGTT } \\
\text { R: AGGATCCTCTCTGGCTCCAT }\end{array}$ \\
\hline BAG3 ${ }^{C 151 R}$ sequencing & $\begin{array}{l}\text { F: TTGGCCAAATAGCAACCTCT } \\
\text { R: AGATGCCCTGCATGTGAAC }\end{array}$ \\
\hline
\end{tabular}

\section{Western blot and capillary immunoassay}

461 Cells were lysed on-plate by adding RIPA buffer to the plate, incubating on ice for 46230 minutes and scraping. Then lysates were harvested, clarified by centrifugation for 10 463 minutes and quantified using a microBCA Protein Assay Kit (Thermo Scientific). For 464 membrane based western blot, procedure was described previously ${ }^{25}$. Bands were 
analyzed and quantified using the ImageStudio Software (Li-Cor). All protein intensities were normalized by a housekeeping gene loading control (GAPDH). Secondary antibodies were Goat Anti-Mouse IRDye 680LT (dilution 1:20000) and Donkey AntiRabbit IgG IRDye (dilution 1:10000) (both by Li-Cor). For capillary immunoassay, the Simple Western Wes platform (ProteinSimple) was used following the protocol from the provider. Briefly, $1 \mu \mathrm{L}$ of protein was diluted with Sample Buffer and mixed with Fluorescent Master Mix and denatured for 5 minutes at $95^{\circ} \mathrm{C}$. For secondary antibodies, a mix of anti-rabbit and anti-mouse solutions (1:1) Secondary HRP Antibodies was used. Then all solutions were loaded on a capillary cartridge as per manufacturer's instructions. Band intensities were quantified using Compass software (ProteinSimple) and values were normalized by GAPDH as a loading control. Primary antibodies used in this study: Anti-BAG3 rabbit polyclonal (ProteinTech, 10599-9-Ap), Monoclonal ANTIFLAG M2 (Sigma-Aldrich, F1804), polyclonal Anti-GAPDH rabbit (Abcam, ab9458), antiHSPB8 (Abcam, ab96837).

\section{HEK293T cell culture, protein overexpression and APMS} (high glucose, L-glutamine, sodium pyruvate; Gibco) supplemented with $10 \%$ Hyclone 483 Fetal Bovine Serum (GE Life Sciences), GlutaMax (Gibco) and 1-00U/ml of 484 Penicillin/Streptomycin (Gibco). Cells were passaged using $0.05 \%$ Trypsin solution 485 (Gibco) whenever necessary. Plasmids expressing BAG3 variants were generated from 486 BAG3 cDNA, cloned into an expression plasmid driven by CMV promoter and modified 487 using QuikChange Site-Directed Mutagenesis Kit (Agilent). For each affinity purification, 488 a $\sim 80 \%$ confluent $15 \mathrm{~cm}^{2}$ cell culture dish was transfected with $10 \mu \mathrm{g}$ of plasmid using 489 the PolyJet In Vitro DNA Transfection Reagent (SignaGen) following provider's 490 instructions. For controls, we used cells transfected with empty vector or a plasmid 491 expressing the 3xFLAG peptide. Two days later, cells were harvested using Phosphate 492 Buffer saline (PBS) with 10mM EDTA, washed twice with PBS, and resuspended in $4931 \mathrm{~mL}$ of lysis buffer (50 mM Tris-HCl pH 7.5, $150 \mathrm{mM} \mathrm{NaCl}, 0.5 \mathrm{mM}$ EDTA, 0.5\% NP-40 494 and 1x cOmplete protease inhibitor cocktail (Roche)). Samples were lysed by rotating at $4954^{\circ} \mathrm{C}$ for 30 minutes, and cleared by centrifugation. For binding, the lysates were added 
496 FLAG M2 Magnetic Bead (Sigma-Aldrich; $30 \mu \mathrm{L}$ of bead slurry per reaction) and allowed

497 to bind $4^{\circ} \mathrm{C}$ for 2 hours. Beads were then collected using a magnetic stand and washed

4983 times with wash buffer (50 mM Tris-HCl, pH 7.5, NaCl 150 mM, EDTA 0.5 mM,

499 supplemented with $0.05 \%$ NP-40), with an extra wash with no NP-40. Then, beads were

500 resuspended in $30 \mu \mathrm{L}$ of elution buffer (1\% Rapigest SF Surfactant (Waters), $5 \mathrm{mg} / \mathrm{ml}$

501 3xFLAG peptide (synthesized by Elim Biopharmaceuticals Inc), in wash buffer without

502 NP-40) and eluted at room temperature for 30 minutes with constant shaking.

503

504

Eluted protein samples were reduced with DTT $(2.5 \mathrm{mM})$ at $50^{\circ} \mathrm{C}$ for 30 minutes,

505 and alkylated with iodoacetamide $(2.5 \mathrm{mM})$ for 40 minutes at room temperature in the

506 dark. After that, $0.5 \mu \mathrm{g}$ of sequencing grade trypsin (Promega) was added to the sample

507 and incubated overnight at $37^{\circ} \mathrm{C}$. Peptides were desalted and concentrated on ZipTip

508 C18 pipette tips (Millipore) according to the manufacturer's protocol. Digested peptides

509 were resuspended in 0.1\% Formic Acid and analyzed by LC-MS/MS on a Velos Pro ion

510 trap mass spectrometer (Thermo Scientific) system equipped with an Easy-nLC 1000

511 ultra high pressure liquid chromatography and autosampler system (Thermo Scientific).

512 Samples were injected onto a pre-column (2 cm x $100 \mu \mathrm{m}$ I.D. packed with ReproSil Pur

$513 \mathrm{C} 18 \mathrm{AQ} 5 \mu \mathrm{m}$ particles) in 0.1\% formic acid and then separated with a two-hour gradient

514 from $5 \%$ to $30 \%$ ACN in $0.1 \%$ formic acid on an analytical column (10 $\mathrm{cm} \times 75 \mu \mathrm{m}$ I.D.

515 packed with ReproSil Pur C18 AQ $3 \mu \mathrm{m}$ particles). Each full scan was followed by 20

516 collision-induced dissociation MS/MS scans of the 20 most intense peaks. Dynamic

517 exclusion was enabled for 30 seconds. Raw data files were converted into peak lists

518 using PAVA software ${ }^{64}$. Spectra were then searched using Protein Prospector 5.10.1

519 (http://prospector.ucsf.edu/) using the SwissProt database of human proteins (April

520 2012). One missing cleavage was allowed. Fragment mass tolerance was set as $0.8 \mathrm{Da}$

521 and parent mass tolerance as $1 \mathrm{Da}$. As for modifications, carbamidomethylation of

522 cysteines was set as constant and acetylation of protein N-termini and methionine

523 oxidation and methionine loss at $\mathrm{N}$-termini were set as variable. The results from

524 Protein Prospector were further filtered as follows: minimum Protein Score of 22.0,

525 minimum Peptide Score of 15.0, maximum Protein E-Value of 0.01 and maximum

526 Peptide E-Value of 0.05 . All sample runs were manually curated for good values of bait 
527 count, total spectral counts and total number of unique proteins identified, leaving a total 528 of 5 replicates per bait for downstream analysis. To filter out sample carryover across 529 runs, we discarded each protein entry with less than half the spectral counts of the 530 previous runs and that was present in less than $30 \%$ of all the experiments, similar to 531 what others have described ${ }^{65}$. To deal with non-unique peptides, we took only the first 532 protein with the most unique peptides and any other protein in the group that had at 533 least one unique peptide across replicates. Spectral count values were then normalized 534 to equate BAG3 counts (except for the truncation variants) and SAINTexpress v3.6.34 $535{ }^{66}$ was used with the sample compression option turned on to 4 samples ('-R4') for the 536 statistical analysis of interactors. Control experiments were not compressed to minimize 537 the impact of unremoved carryover. The scoring was performed independently for each 538 bait condition. A database containing all GO terms with less than 20 members was input 539 to boost scores of known interactors ${ }^{66}$. A final FDR cutoff of 0.1 was used. The dot plot 540 for visualization of results was generated using the ProHits-Viz suite ${ }^{67}$. Cytoscape ${ }^{68}$ 541 version 3.4.0 was used for the network graph generation, with the CORUM ${ }^{69}$ database 542 (version 3.0) used for interconnecting the nodes. Output from SAINTexpress analyses 543 of BAG3 variants expressed in HEK293T cells can be found in Table S1.

\section{Immunoprecipitation of iPS/iPS-CM samples and unbiased proteomics analysis}

$546 \quad$ At day 30 of differentiation, enriched iPS-CM cultures were harvested by

547 scraping on ice-cold PBS, washed twice with ice-cold PBS and flash-frozen in dry ice

548 with ethanol. For the bortezomib treatment condition, bortezomib was added to the 549 media to $100 \mu \mathrm{M}$ final concentration 24 hours prior to the harvesting. For iPS-CM

550 APMS, 25-30 million cells per sample were used. For iPSC APMS, three 90\%

551 confluent $15 \mathrm{~cm}$ dishes were used per sample. As a negative control for nonspecific

552 binding, WTC iPSC or iPS-CM cells not expressing any 3xFLAG affinity epitope were

553 used. All the experimental conditions were performed in four replicates, each one on a

554 different day. For each replicate all the different conditions were run in parallel. For

555 mass spectrometry, all samples were run sequentially. Cell pellets were thoroughly

556 resuspended in lysis buffer (0.1\% NP-40, $300 \mathrm{mM} \mathrm{NaCl}, 20 \%$ Glycerol, $2 \mathrm{mM} \mathrm{MgCl}$,

$557 \quad 0.5$ mM EDTA, 0.5 mM EGTA, 1 mM PMSF, 1 mM DDT in 50 mM HEPES-NaOH pH 
558 8.0, supplemented with complete protease inhibitor cocktail (Roche) and Benzonase 559 Nuclease $(50 \mathrm{U} / \mathrm{ml}$, Sigma-Aldrich)). Cells were lysed by four flash freeze-thaw cycles, 560 followed by incubation for $20 \mathrm{~min}$ at $4^{\circ} \mathrm{C}$ in constant rotation. After clearing lysates by 561 centrifugation, protein extracts were diluted three-fold (in 0.5 mM EDTA, 0.5 mM EGTA, $5621 \mathrm{mM}$ PMSF in 50mM HEPES-NaOH pH 8.0) to reduce salt content and incubated with $56330 \mu \mathrm{L}$ anti-FLAG M2 Magnetic Beads slurry (Sigma-Aldrich) for 2-3 hours. Beads were 564 then rinsed in wash buffer (3x washes in 0.01\% NP-40, 1 mM PMSF, 0.5\% EDTA, 0.5\% 565 EGTA in 50 mM HEPES, pH 8.0; 1x wash on buffer without NP-40). Beads loaded with FLAG-enriched proteins were reduced (5 mM TCEP), alkylated (15 mM iodoacetamide),

567 and digested with 1\% (w/v) trypsin overnight. Resulting peptides were desalted by

568 OMIX C18 desalting tips (Agilent) following protocol by provider and dried on a speed569 vac. Peptides were resuspended in $0.1 \%$ formic acid and an iRT peptide standard mix (Biognosys) was spiked in prior to use for mass spectrometry.

571

For unbiased (non-targeted) analysis of pulldown samples, peptides were

573 analyzed by liquid chromatography tandem mass spectrometry (LC MS/MS) with an

574 Easy-nLC 1000 (Thermo Fisher, San Jose, CA) coupled to an Orbitrap Fusion Tribrid 575 Mass Spectrometer (Thermo Fisher Scientific, San Jose, CA). Online LC separation 576 was carried out using a $75 \mu \mathrm{m} \times 25 \mathrm{~cm}$ fused silica IntregraFrit capillary column (New 577 Objective, Woburn, MA) packed in-house with 1.9 $\mu \mathrm{m}$ Reprosil-Pur C18 AQ reverse578 phase resin (Dr. Maisch-GmbH). Peptides were eluted at a flowrate of $300 \mathrm{~nL} / \mathrm{min}$ using 579 a linear gradient of 5-30\% B in $45 \mathrm{~min}$, and 30-95\% B for $25 \mathrm{~min}$ (mobile phase buffer 580 A: $0.1 \%$ formic acid; mobile phase buffer B: $0.1 \%$ formic acid in ACN). Survey scans of 581 peptide precursors from 400 to $1600 \mathrm{~m} / \mathrm{z}$ were performed at $120 \mathrm{~K}$ resolution in the 582 Orbitrap, with an AGC target of $2 \times 105$, and a maximum injection time of $100 \mathrm{~ms}$.

583 Tandem MS (MS2) was performed by isolation with the quadrupole, HCD fragmentation 584 with normalized collision energy of $30 \%$, and rapid scan MS analysis in the ion trap. The 585 MS2 ion count target was set to $10^{4}$ and the max injection time was $35 \mathrm{~ms}$. Precursors 586 with charge state 2-7 were sampled for MS2 and dynamically excluded for $20 \mathrm{~s}$ 587 (tolerance of 10 ppm). Monoisotopic precursor selection was turned on, and the 588 instrument was run in top speed mode with 3-s cycles. For protein identification and 
589 quantification, MaxQuant software v1.5.3.30 was used ${ }^{70}$. Tandem mass spectrometry

590 (MS/MS) spectra were searched against the November 2016 release of the UniProt

591 complete human proteome sequence database. MaxQuant was run on default

592 parameters, allowing for 2 maximum missed cleavages, with a first search peptide

593 tolerance of $20 \mathrm{ppm}$ and a main search peptide tolerance of $4.5 \mathrm{ppm}$. Methionine

594 oxidation and $\mathrm{N}$-terminal acetylation were set as variable modifications, and

595 carbamidomethylation of cysteines as fixed modification. The 'match between runs'

596 setting was activated (window of $0.7 \mathrm{~min}$ ) to improve peptide identification. Mass

597 spectrometry raw data and search results files have been deposited to the

598 ProteomeXchange Consortium via the PRIDE partner repository ${ }^{71}$.

599

600

Peptide sequences were used to validate pulldown of intended BAG3 variants.

601 Proteins with two or fewer peptides identified were discarded, as were typical common

602 contaminant proteins (downloaded from http://maxquant.org/). Intensity values were

603 normalized by median equalization of the peptide lists, and aggregated into protein

604 intensity values. SAINTq v0.0.4 ${ }^{72}$ was then used to compare each experimental

605 condition to the no FLAG control set individually. Significant putative protein interactors

606 were selected with a Bayesian False Discovery Rate (FDR) cutoff of 0.1. Euler

607 diagrams were generated using BioVenn ${ }^{73}$ or the R package eulerr ${ }^{74}$.

608

Targeted proteomics follow-up of iPS-CM APMS high confidence protein-protein

610 interactions

611 A list of 118 proteins from our unbiased APMS study was chosen for targeted

612 proteomics quantitation (43 proteins significant in our iPS-CM APMS studies, 37

613 significant in iPSC or HEK293T cell APMS, 35 nonsignificant but previously described

614 as interacting with or related to BAG3, 3 low-variability controls, plus BAG3 and the iRT

615 peptides). To increase the number of peptides used for quantitation of these proteins,

616 we downloaded extra peptide sequences and iRT standardized retention time values

617 from SRMatlas ${ }^{75}$ (www.mrmatlas.com). The isolation list of all peptides (empirical +

618 predicted) was then used to perform a preliminary scan of a pooled sample from all 
619 conditions using a Q-Exactive Plus Mass Spectrometer (Thermo Fisher). Skyline v4.276 620 was used to manually select best peptides and fragments based on ppm, q-value and 621 uniqueness to protein target. The final isolation list contained 4 peptides per protein

622 (except for BAG3, which kept 8 peptides, and the 11 iRT peptides; Table S3) and was

623 split into two acquisition methods to minimize the number of concurrent peptides per 624 window. For targeted quantitation of peptide abundance, we used a Parallel Reaction

625 Monitoring ${ }^{77}$ method on a Thermo Fisher Q Exactive. Online LC separation was carried

626 out using a $75 \mu \mathrm{m}$ x $25 \mathrm{~cm}$ fused silica PicoTip capillary column (New Objective,

627 Woburn, MA) packed in-house with $1.9 \mu \mathrm{m}$ Reprosil-Pur C18 AQ reverse-phase resin

628 (Dr. Maisch-GmbH). Peptides were eluted at a flowrate of $300 \mathrm{~nL} / \mathrm{min}$ using a linear

629 gradient of $7-36 \% \mathrm{~B}$ in $52 \mathrm{~min}$, and 36-85\% B for $7 \mathrm{~min}$ (mobile phase buffer A: 0.1\%

630 formic acid; mobile phase buffer B: $0.1 \%$ formic acid in $80 \%$ ACN). Peptides were

631 directly injected into the mass spectrometer in positive ion mode over the course of a 75

632 min total acquisition time. MS2 scan were acquired in profile mode for peptides defined

633 on the inclusion list at a resolution of $17.5 \mathrm{~K}$, with a $2 \times 10^{5} \mathrm{AGC}$ target, $30 \mathrm{~ms}$ maximum

634 injection time, a loop count of 40 , an MSX count of 1 , a $2 \mathrm{~m} / \mathrm{z}$ isolation window, and an

635 NCE of 27.

Raw data was loaded into Skyline to manually select best peptides and

638 boundaries, ending on a total of 113 proteins with at least 2 good peptides. MSStats ${ }^{78}$

639 was then used to format the data and obtain a matrix of log intensities per sample.

640 Missing data ( $0.7 \%$ of total data) was imputed by averaging remaining replicates for the

641 same protein and condition. For analysis of the data, we used a hypervariate linear

642 model analysis followed by empirical bayes testing to obtain a robust estimation of

643 differential protein abundance across samples, while controlling for replicate batch

644 variability. For this we used the limma package in $\mathrm{R}^{79,80}$. First, to obtain a list of high-

645 confidence interactions for each condition, we fit a model for condition vs no-FLAG

646 control. Then, a second model was fit to compare the intensities for all significant hits in

647 the BAG3 ${ }^{\mathrm{C} 151 \mathrm{R}}$, BAG3 ${ }^{\mathrm{E} 455 \mathrm{~K}}$ or BAG3 (+bortezomib) conditions against BAG3 ${ }^{\mathrm{WT}}$. Adjusted

648 p-value threshold was set at 0.05 in both steps. Mass spectrometry raw data and 
649 Skyline files have been deposited to the ProteomeXchange Consortium using the

650 Panorama Public partner repository81 (link: https://panoramaweb.org/28ZWVY.url).

652 Immunofluorescence staining of iPS-CM and imaging

653 For immunostaining of iPS-CM in culture, cells were washed with PBS and fixed

654 with 4\% Paraformaldehyde at room temperature for 20 minutes. Then cells were 655 washed three times with PBS supplemented with 0.1\% Triton X-100 (PBS-Triton) and 656 incubated with 5\% Bovine Albumin Serum in PBS-Triton for 1 hour at room temperature 657 to block and permeabilize. Then cells were incubated overnight at $4^{\circ} \mathrm{C}$ with a solution of 658 primary antibody in PBS-Triton. The morning after, cells were washed three times with 659 PBS-Triton and then incubated in secondary antibodies in PBS-Triton for 1 hour at room 660 temperature. Cells were washed three more times with PBS-Triton (first wash

661 supplemented with 1:1000 DAPI) and kept in PBS supplemented with 0.02\% Sodium 662 Azide (Sigma-Aldrich) until imaging. Secondary antibodies used were Goat Anti-Rabbit 663 Alexa Fluor 594 and Goat Anti-Mouse IgG Alexa Fluor 488 (both at a 1:500 dilution; 664 both from Thermo Fisher Scientific). For primary antibodies, we used rabbit polyclonal 665 anti-BAG3 (Protein Tech, 10599-1-A), mouse monoclonal anti-FLAG M2 (Sigma666 Aldrich, F1804), and mouse monoclonal anti-MYBPC3 (Santa Cruz Biotechnology, sc667 137180), all at a 1:200 dilution. Images used for the unbiased analysis of sarcomere 668 structure were acquired using an ArrayScan VTI HCS Reader (ThermoFisher) at a 10x 669 magnification. Images used for high-magnification figures in this manuscript were 670 acquired using an ImageXpress Micro Confocal High-Content Imaging System 671 (Molecular Devices). Raw images were processed using the R package EBImage ${ }^{82}$ or 672 Image ${ }^{83}$ for visualization and figure preparation.

674 Gene knockdown on iPS-CM and unbiased image analysis

675 For quality control and protocol optimization experiments, FlexiTube siRNA 676 (Qiagen) oligonucleotides against BAG3 or a scramble control were used. For imaging 677 screening and validation experiments, a Silencer Select siRNA Custom Library 678 (Ambion) was used. Gene targets were selected for being BAG3 iPS-CM interactor in 
679 our AP-MS experiments, being a prominent BAG3 interactor in the literature, or being 680 an important myofibrillar/z-disk component or chaperone complex. For all gene targets, 681 three different siRNA oligos were combined. High purity iPS-CM cultures (iCell 682 Cardiomyocytes ${ }^{2}$, Lot\#CMC331743, >99\% cTnT+) were obtained from Cellular 683 Dynamics International (Madison, WI). Vials were thawed as per manufacturer 684 instructions and seeded on 24 well plates (for quality control analyses) or Falcon 96-well 685 Black/Clear Imaging Microplate (Corning) at a density of $\sim 53,000$ cells $/ \mathrm{cm}^{2}$. Cells were 686 allowed to recover for five days and transfected with $1 \mu \mathrm{M}$ total siRNA using 687 Lipofectamine RNAiMAX Transfection Reagent (ThermoFisher) $(0.05 \mu \mathrm{L}$ or 0.25

$688 \mu \mathrm{L} /$ well, respectively) following protocol from provider. Cells were left in the siRNA 689 solution for 48 hours, and then media was changed for fresh cardiomyocyte 690 maintenance media as usual. Cells were used for subsequent analyses (harvesting or 691 immunostaining) 10 days after transfection.

692 For the unbiased analysis of sarcomere staining images on iPS-CM gene 693 knockdowns, three biological replicates were performed for each siRNA condition.

694 Screening plates included internal Scramble and BAG3 control conditions. In parallel, 695 additional wells were prepared for each one of the BAG3 and Scramble siRNA 696 conditions for training (32 wells) and validation (8 wells) of the model. Nine images per 697 well were used in all conditions. Images were used to train a supervised machine 698 learning image classification model using PhenoLearn framework

699 (www.phenolearn.com), as described elsewhere ${ }^{41,42}$. This model was used to generate 700 a classification score for each image from the screening plates indicating the similarity 701 to the BAG3 siRNA (score closer to 0) or Scramble siRNA (score closer to 1) training 702 sets. For the MYBPC3 staining images, this classification score was dubbed "BAG3 703 Sarcomere Score". The median score for each well was used for further analyses.

704 Images from corner wells were discarded due to loss of viability, and conditions with 705 less than 3 replicates were discarded. The R package EBImage ${ }^{82}$ was used for the 706 quantification of cell viability (nuclei count) and BAG3 staining intensities. Statistical 707 significance for the differences across conditions was performed using a One-Way 708 ANOVA with post-hoc multiple comparisons corrected by Dunnett test. Information on 
709 gene targets selected, siRNA sequences used and image analysis summary values can

710 be found in Table S2.

\section{Bortezomib dose-response curve}

713 Day 30 frozen iPS-CM vials for all the cell lines used were thawed (as described

714 above), counted and seeded directly into 96-well plates. All lines were seeded in parallel

715 at three different densities (15,000, 30,000, and 60,000 cells/well), each in triplicate.

716 Cells were allowed to recover for 5 days with RPMI/B27+ media changes every other

717 day. On day 5, media was supplemented with the desired concentration of bortezomib

718 (Sigma Aldrich) in DMSO, with the equivalent volume of DMSO for the vehicle-only

719 controls. Cells were incubated for 48 hours and then wells were washed twice with PBS

720 before adding fresh RPMI/B27+ media. Seven days later, cells were treated with

721 RPMI/B27+ with 10\% resazurin blue reagent (PrestoBlue, Thermo Scientific) for 1 hour

722 at $37^{\circ} \mathrm{C}$. Fluorescence was then read for each well using a SpectraMax i3 plate reader

723 (Molecular Devices) according to the provider's instructions. For each cell line, the

724 seeding density that resulted in the most comparable viability readout across lines for

725 the DMSO-only condition was chosen for the calculations. Viability readout values were

726 normalized to the range from 0 (media only) to 1 (vehicle only) and fit on a two-

727 parameter log-logistic dose-response model using the R package $d r c^{84}$. Confidence

728 intervals were adjusted to control for family-wise error rates using the package

729 multcomp ${ }^{85}$. Pairwise EC50 comparisons were then performed using a One-way

730 ANOVA test with post hoc Zidak correction using Graphpad Prism (version 8.4.3.686 for

731 Windows, GraphPad Software, San Diego, California USA, www.graphpad.com). 


\section{Acknowledgements}

We thank the Gladstone Stem Cell Core and the Gladstone Assay Development

735 and Drug Discovery Core for providing their technical support and experimental

736 expertise. We also would like to thank Dr. Reuben Thomas from the Gladstone

737 Bioinformatics core for his advice on data analysis. We are also very grateful to Dr. Jeff

738 Johnson, Dr. Ruth Huttenhain and Dr. Gwendolyn Jang for their advice on affinity

739 purification and mass spectrometry experiments. We thank Prof. Jason Gestwicki and

740 their team for their scientific and technical advice. We thank Amanda Chan, Alisha Birk,

741 Edward Shin, Chiara Marley and Serah Kang for their technical support. We also thank

742 Francoise Chanut from Gladstone Editorial Services for her feedback on manuscript

743 preparation.

\section{Funding}

J.A.P.B. was supported by a Graduate Fellowship from Fundación "La Caixa" (ID

747 100010434, \#LCF/BQ/US10/10230024), a Bristol-Myers Squibb PCO Graduate

748 Fellowship for Assessing Early Drug Liabilities (ID No. 63376), and a Predoctoral

749 Fellowship from the American Heart Association (15PRE2570008507 and

750 13PRE1612001307). B.R.C. was supported by the National Institutes of Health (R01-

751 HL130533, R01-HL13535801, P01-HL146366) and by funding from Tenaya

752 Therapeutics. B.R.C. acknowledges support through a gift from the Roddenberry

753 Foundation and Pauline and Thomas Tusher. N.J.K. was supported by P01 HL146366.

754 R.M.K. was supported by NIH fellowship F32Al127291. L.M.J. was supported by a

755 postdoctoral fellowship from the California Institute of Regenerative Medicine (CIRM;

756 TG2-01160) and a Career Development Award from the National Institute of Child

757 Health and Development (1K12HD072222).

760 B.R.C. is a founder of Tenaya Therapeutics (www.tenayatherapeutics.com), a 
762 cardiomyopathies. B.R.C. and J.J.H. hold equity in Tenaya. The Krogan Laboratory has

763 received research support from Vir Biotechnology and F. Hoffmann-La Roche. N.J.K. has

764 a consulting agreements with the Icahn School of Medicine at Mount Sinai, New York. He

765 is a shareholder of Tenaya Therapeutics, Maze Therapeutics and Interline Therapeutics.

\section{Author Contributions}

767 J.A.P.B., L.M.J., P.S., N.J.K., and B.R.C. designed and supervised the study.

768 J.P.B., C.L.J., A.T., J.J.H., W.V.R. and K.W. performed cell line generation, cell culture

769 and differentiation. J.P.B., R.M.K, E.P. and D.L.S. performed affinity purification-mass

770 spectrometry experiments and analyses. J.P.B., K.W. and M.A.M. performed siRNA

771 knockdown panel and image analysis. J.P.B. and L.M.J. performed bortezomib toxicity

772 assay and analyses. All authors contributed to writing the manuscript and preparing the

773 figures. 


\section{Bibliography}

776 1. Virani, S. S. et al. Heart Disease and Stroke Statistics-2021 Update: A Report From the 777 American Heart Association. Circulation 143, e254-e743 (2021).

778 2. Burke, M. A., Cook, S. A., Seidman, J. G. \& Seidman, C. E. Clinical and Mechanistic

779 Insights Into the Genetics of Cardiomyopathy. J. Am. Coll. Cardiol. 68, 2871-2886 (2016).

780 3. Weintraub, R. G., Semsarian, C. \& Macdonald, P. Dilated cardiomyopathy. Lancet 390, 781 400-414 (2017).

782 4. Harper, A. R., Nayee, S. \& Topol, E. J. Protective alleles and modifier variants in human health and disease. Nat. Rev. Genet. 16, 689-701 (2015).

784 5. Villard, E. et al. A genome-wide association study identifies two loci associated with heart failure due to dilated cardiomyopathy. Eur. Heart J. 32, 1065-1076 (2011).

6. Esslinger, U. et al. Exome-wide association study reveals novel susceptibility genes to sporadic dilated cardiomyopathy. PLoS One 12, e0172995 (2017).

7. Aragam, K. G. et al. Phenotypic refinement of heart failure in a national biobank facilitates genetic discovery. Circulation (2018). doi:10.1161/CIRCULATIONAHA.118.035774

8. Shah, S. et al. Genome-wide association and Mendelian randomisation analysis provide insights into the pathogenesis of heart failure. Nat. Commun. 11, 163 (2020).

9. Choquet, H. et al. Meta-Analysis of 26638 Individuals Identifies Two Genetic Loci Associated With Left Ventricular Ejection Fraction. Circ. Genom. Precis. Med. 13, e002804 (2020).

795 10. Verweij, N. et al. The genetic makeup of the electrocardiogram. Cell Syst. 11, 229-238.e5 (2020).

11. de Denus, S. et al. A genetic association study of heart failure: more evidence for the role of BAG3 in idiopathic dilated cardiomyopathy. ESC Heart Fail. (2020). doi:10.1002/ehf2.12934

12. Garnier, S. et al. Genome-wide association analysis in dilated cardiomyopathy reveals two new players in systolic heart failure on chromosomes 3p25.1 and 22q11.23. Eur. Heart J. 42, 2000-2011 (2021).

803 13. Aung, N. et al. Genome-Wide Analysis of Left Ventricular Image-Derived Phenotypes

804 Identifies Fourteen Loci Associated With Cardiac Morphogenesis and Heart Failure 
14. Franaszczyk, M. et al. The BAG3 gene variants in Polish patients with dilated cardiomyopathy: four novel mutations and a genotype-phenotype correlation. J. Transl. Med. 12, 192 (2014).

15. Norton, N. et al. Genome-wide studies of copy number variation and exome sequencing identify rare variants in BAG3 as a cause of dilated cardiomyopathy. Am. J. Hum. Genet. 88, 273-282 (2011).

16. Feldman, A. M. et al. Decreased levels of BAG3 in a family with a rare variant and in idiopathic dilated cardiomyopathy. J. Cell Physiol. 229, 1697-1702 (2014).

17. Chami, N. et al. Nonsense mutations in BAG3 are associated with early-onset dilated cardiomyopathy in French Canadians. Can. J. Cardiol. 30, 1655-1661 (2014).

18. Citro, R. et al. Polymorphisms of the antiapoptotic protein bag3 may play a role in the pathogenesis of tako-tsubo cardiomyopathy. Int. J. Cardiol. 168, 1663-1665 (2013).

19. Homma, S. et al. BAG3 deficiency results in fulminant myopathy and early lethality. Am. J. Pathol. 169, 761-773 (2006).

20. Domínguez, F. et al. Dilated Cardiomyopathy Due to BLC2-Associated

822 21. Behl, C. Breaking BAG: The Co-Chaperone BAG3 in Health and Disease. Trends Pharmacol. Sci. 37, 672-688 (2016).

824 22. Knezevic, T. et al. BAG3: a new player in the heart failure paradigm. Heart Fail. Rev. 20, $825 \quad 423-434(2015)$.

826 23. Gamerdinger, M., Carra, S. \& Behl, C. Emerging roles of molecular chaperones and cochaperones in selective autophagy: focus on BAG proteins. J. Mol. Med. 89, 1175-1182 (2011).

24. Martin, T. G. \& Kirk, J. A. Under construction: The dynamic assembly, maintenance, and

831 25. Judge, L. M. et al. A BAG3 chaperone complex maintains cardiomyocyte function during 832 proteotoxic stress. JCI Insight (2017).

833 26. Ulbricht, A., Arndt, V. \& Höhfeld, J. Chaperone-assisted proteostasis is essential for 834 mechanotransduction in mammalian cells. Commun Integr Biol 6, e24925 (2013).

835 27. Fang, X. et al. Loss-of-function mutations in co-chaperone BAG3 destabilize small HSPs 836 and cause cardiomyopathy. J. Clin. Invest. (2017). 
28. Martin, T. G. et al. Cardiomyocyte contractile impairment in heart failure results from reduced BAG3-mediated sarcomeric protein turnover. Nat. Commun. 12, 2942 (2021).

29. Meister-Broekema, M. et al. Myopathy associated BAG3 mutations lead to protein aggregation by stalling Hsp70 networks. Nat. Commun. 9, 5342 (2018).

30. Lo Sardo, V. et al. Unveiling the Role of the Most Impactful Cardiovascular Risk Locus through Haplotype Editing. Cell 175, 1796-1810.e20 (2018).

843 31. Kishore, S. et al. A Non-Coding Disease Modifier of Pancreatic Agenesis Identified by Genetic Correction in a Patient-Derived iPSC Line. Cell Stem Cell 27, 137-146.e6 (2020).

32. Wang, G. et al. Modeling the mitochondrial cardiomyopathy of Barth syndrome with induced pluripotent stem cell and heart-on-chip technologies. Nat. Med. 20, 616-623 (2014).

33. Warren, C. R. et al. Induced pluripotent stem cell differentiation enables functional validation of GWAS variants in metabolic disease. Cell Stem Cell 20, 547-557.e7 (2017).

34. McDermott-Roe, C. et al. Investigation of a dilated cardiomyopathy-associated variant in $B A G 3$ using genome-edited iPSC-derived cardiomyocytes. JCI Insight (2019).

35. Ganassi, M. et al. A Surveillance Function of the HSPB8-BAG3-HSP70 Chaperone Complex Ensures Stress Granule Integrity and Dynamism. Mol. Cell 63, 796-810 (2016).

36. Nowis, D. et al. Cardiotoxicity of the anticancer therapeutic agent bortezomib. Am. J. Pathol. 176, 2658-2668 (2010).

37. Orciuolo, E. et al. Unexpected cardiotoxicity in haematological bortezomib treated patients. Br. J. Haematol. 138, 396-397 (2007).

38. Grandin, E. W., Ky, B., Cornell, R. F., Carver, J. \& Lenihan, D. J. Patterns of cardiac toxicity associated with irreversible proteasome inhibition in the treatment of multiple myeloma. J Card Fail 21, 138-144 (2015).

39. Kieserman, J. M., Myers, V. D., Dubey, P., Cheung, J. Y. \& Feldman, A. M. Current landscape of heart failure gene therapy. J. Am. Heart Assoc. 8, e012239 (2019).

40. Knezevic, T. et al. Adeno-associated Virus Serotype 9 - Driven Expression of BAG3 Improves Left Ventricular Function in Murine Hearts with Left Ventricular Dysfunction Secondary to a Myocardial Infarction. JACC Basic Transl. Sci. 1, 647-656 (2016).

41. Maddah, M. et al. A non-invasive platform for functional characterization of stem-cellderived cardiomyocytes with applications in cardiotoxicity testing. Stem Cell Rep. 4, 621$631(2015)$. 
42. Grafton, F. et al. Deep Learning Predicts Patterns of Cardiotoxicity in a High-Content Screen Using Induced Pluripotent Stem Cell-Derived Cardiomyocytes. BioRxiv (2021). doi:10.1101/2021.03.23.436666

43. Schroer, A., Pardon, G., Castillo, E., Blair, C. \& Pruitt, B. Engineering hiPSC cardiomyocyte in vitro model systems for functional and structural assessment. Prog. Biophys. Mol. Biol. 144, 3-15 (2019).

874

44. van Mil, A. et al. Modelling inherited cardiac disease using human induced pluripotent stem cell-derived cardiomyocytes: progress, pitfalls, and potential. Cardiovasc. Res. 114, 18281842 (2018).

878

45. Musunuru, K. et al. Induced pluripotent stem cells for cardiovascular disease modeling and precision medicine: A scientific statement from the american heart association. Circ. Genom. Precis. Med. 11, e000043 (2018).

46. Kane, R. C., Farrell, A. T., Sridhara, R. \& Pazdur, R. United States Food and Drug Administration approval summary: bortezomib for the treatment of progressive multiple myeloma after one prior therapy. Clin. Cancer Res. 12, 2955-2960 (2006).

47. Métais, A. et al. Asb2 $\alpha$-Filamin A Axis Is Essential for Actin Cytoskeleton Remodeling During Heart Development. Circ. Res. 122, e34-e48 (2018).

48. Mao, Z. \& Nakamura, F. Structure and Function of Filamin C in the Muscle Z-Disc. Int. J. Mol. Sci. 21, (2020).

49. Klimek, C. et al. The Hippo network kinase STK38 contributes to protein homeostasis by inhibiting BAG3-mediated autophagy. Biochim. Biophys. Acta Mol. Cell Res. 1866, 15561566 (2019).

50. Collier, M. P. et al. HspB1 phosphorylation regulates its intramolecular dynamics and mechanosensitive molecular chaperone interaction with filamin C. Sci. Adv. 5, eaav8421

51. Waldt, N. et al. Filamin A Phosphorylation at Serine 2152 by the Serine/Threonine Kinase Ndr2 Controls TCR-Induced LFA-1 Activation in T Cells. Front. Immunol. 9, 2852 (2018). roles of cardiac small heat shock proteins. JCI Insight (2019). 
Int. J. Mol. Sci. 22, (2021).

54. Rauch, J. N. \& Gestwicki, J. E. Binding of human nucleotide exchange factors to heat shock protein 70 (Hsp70) generates functionally distinct complexes in vitro. J. Biol. Chem. 289, 1402-1414 (2014).

903

55. Clarke, L. et al. The international Genome sample resource (IGSR): A worldwide collection of genome variation incorporating the 1000 Genomes Project data. Nucleic Acids Res. 45, D854-D859 (2017).

56. Marcus, J. H. \& Novembre, J. Visualizing the geography of genetic variants. Bioinformatics 33, 594-595 (2017).

57. 1000 Genomes Project Consortium et al. A global reference for human genetic variation. Nature 526, 68-74 (2015).

58. Chang, K. T., Guo, J., di Ronza, A. \& Sardiello, M. Aminode: identification of evolutionary constraints in the human proteome. Sci. Rep. 8, 1357 (2018).

912 59. Mandegar, M. A. et al. CRISPR Interference Efficiently Induces Specific and Reversible 913 Gene Silencing in Human iPSCs. Cell Stem Cell 18, 541-553 (2016).

914 60. Miyaoka, Y. et al. Isolation of single-base genome-edited human iPS cells without antibiotic 915 selection. Nat. Methods 11, 291-293 (2014).

916 61. Hockemeyer, D. et al. Genetic engineering of human pluripotent cells using TALE 917 nucleases. Nat. Biotechnol. 29, 731-734 (2011).

918 62. den Dunnen, J. T. et al. HGVS recommendations for the description of sequence variants:

9192016 update. Hum. Mutat. 37, 564-569 (2016).

920 63. Regan, J. F. et al. A rapid molecular approach for chromosomal phasing. PLoS One 10, $921 \mathrm{e} 0118270(2015)$.

922 64. Guan, S., Price, J. C., Prusiner, S. B., Ghaemmaghami, S. \& Burlingame, A. L. A data 923 processing pipeline for mammalian proteome dynamics studies using stable isotope 924 metabolic labeling. Mol. Cell Proteomics 10, M111.010728 (2011).

925 65. Verschueren, E. et al. Scoring Large-Scale Affinity Purification Mass Spectrometry 926 Datasets with MiST. Curr Protoc Bioinformatics 49, 8.19.1-8.19.16 (2015).

927 66. Teo, G. et al. SAINTexpress: improvements and additional features in Significance Analysis 928 of INTeractome software. J. Proteomics 100, 37-43 (2014).

929 67. Knight, J. D. R. et al. ProHits-viz: a suite of web tools for visualizing interaction proteomics 
data. Nat. Methods 14, 645-646 (2017).

68. Shannon, P. et al. Cytoscape: a software environment for integrated models of biomolecular interaction networks. Genome Res. 13, 2498-2504 (2003).

933 69. Giurgiu, M. et al. CORUM: the comprehensive resource of mammalian protein complexes2019. Nucleic Acids Res. 47, D559-D563 (2019).

935 70. Cox, J. \& Mann, M. MaxQuant enables high peptide identification rates, individualized

936 p.p.b.-range mass accuracies and proteome-wide protein quantification. Nat. Biotechnol. 26, 937 1367-1372 (2008).

938 71. Vizcaíno, J. A. et al. ProteomeXchange provides globally coordinated proteomics data 939 submission and dissemination. Nat. Biotechnol. 32, 223-226 (2014).

940 72. Teo, G. et al. SAINTq: Scoring protein-protein interactions in affinity purification - mass 941 spectrometry experiments with fragment or peptide intensity data. Proteomics 16, 2238$9422245(2016)$.

943 73. Hulsen, T., de Vlieg, J. \& Alkema, W. BioVenn - a web application for the comparison and visualization of biological lists using area-proportional Venn diagrams. BMC Genomics 9,

946 74. Larsson, J. eulerr: Area-Proportional Euler and Venn Diagrams with Ellipses [R package eulerr version 6.1.0]. (2020). at <https://cran.r-project.org/web/packages/eulerr/index.html>

948 75. Kusebauch, U. et al. Human srmatlas: A resource of targeted assays to quantify the complete human proteome. Cell 166, 766-778 (2016).

950 76. Pino, L. K. et al. The Skyline ecosystem: Informatics for quantitative mass spectrometry proteomics. Mass Spectrom Rev 39, 229-244 (2020).

952 77. Peterson, A. C., Russell, J. D., Bailey, D. J., Westphall, M. S. \& Coon, J. J. Parallel reaction monitoring for high resolution and high mass accuracy quantitative, targeted proteomics. Mol. Cell Proteomics 11, 1475-1488 (2012).

955 78. Choi, M. et al. MSstats: an R package for statistical analysis of quantitative mass

956 spectrometry-based proteomic experiments. Bioinformatics 30, 2524-2526 (2014).

957 79. Ritchie, M. E. et al. limma powers differential expression analyses for RNA-sequencing and 958 microarray studies. Nucleic Acids Res. 43, e47 (2015).

959 80. Phipson, B., Lee, S., Majewski, I. J., Alexander, W. S. \& Smyth, G. K. Robust 
961 detect differential expression. Ann Appl Stat 10, 946-963 (2016).

962 81. Sharma, V. et al. Panorama public: A public repository for quantitative data sets processed 963 in skyline. Mol. Cell Proteomics 17, 1239-1244 (2018).

964 82. Pau, G., Fuchs, F., Sklyar, O., Boutros, M. \& Huber, W. EBImage--an R package for image 965 processing with applications to cellular phenotypes. Bioinformatics 26, 979-981 (2010).

966 83. Schindelin, J. et al. Fiji: an open-source platform for biological-image analysis. Nat.

967 Methods 9, 676-682 (2012).

968 84. Ritz, C., Baty, F., Streibig, J. C. \& Gerhard, D. Dose-Response Analysis Using R. PLoS One 969 10, e0146021 (2015).

970 85. Hothorn, T., Bretz, F. \& Westfall, P. Simultaneous inference in general parametric models.

971 Biom J 50, 346-363 (2008).

972 
bioRxiv preprint doi: https://doi.org/10.1101/2021.10.06.463213; this version posted October 6, 2021. The copyright holder for this preprint (which was not certified by peer review) is the author/funder, who has granted bioBxiv a license to display the preprint in perpetuity. It is made A Linkage disequilibrium plotavailable under aCC-BY-ND 4.0 Inter $\mathbf{B}$ tional license.

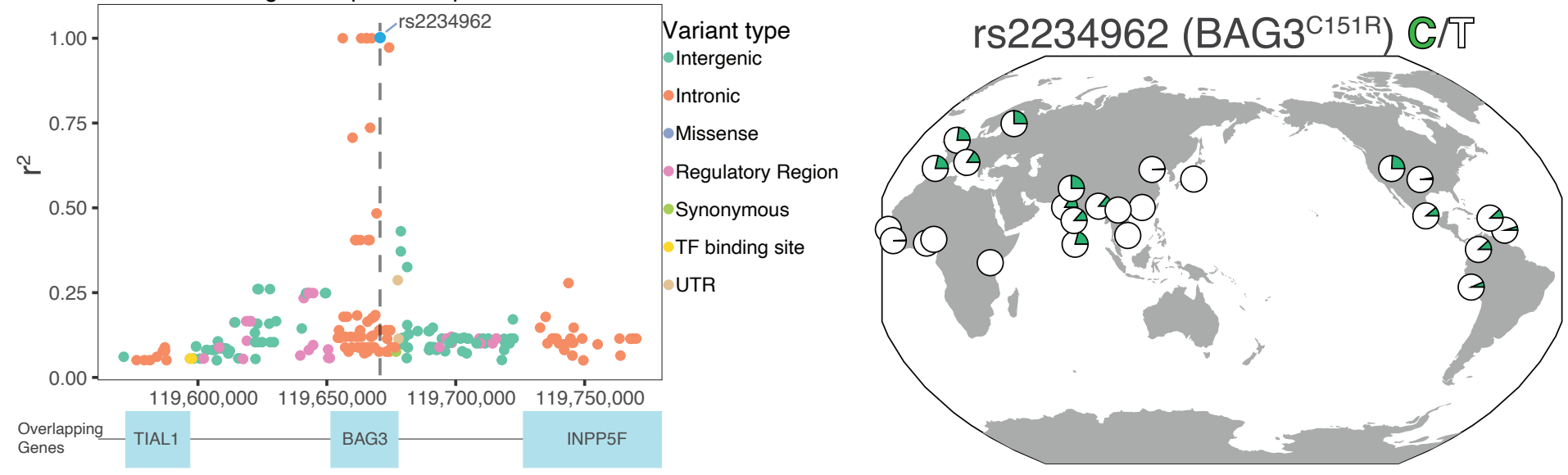

Chromosome 10; 200KB window

C
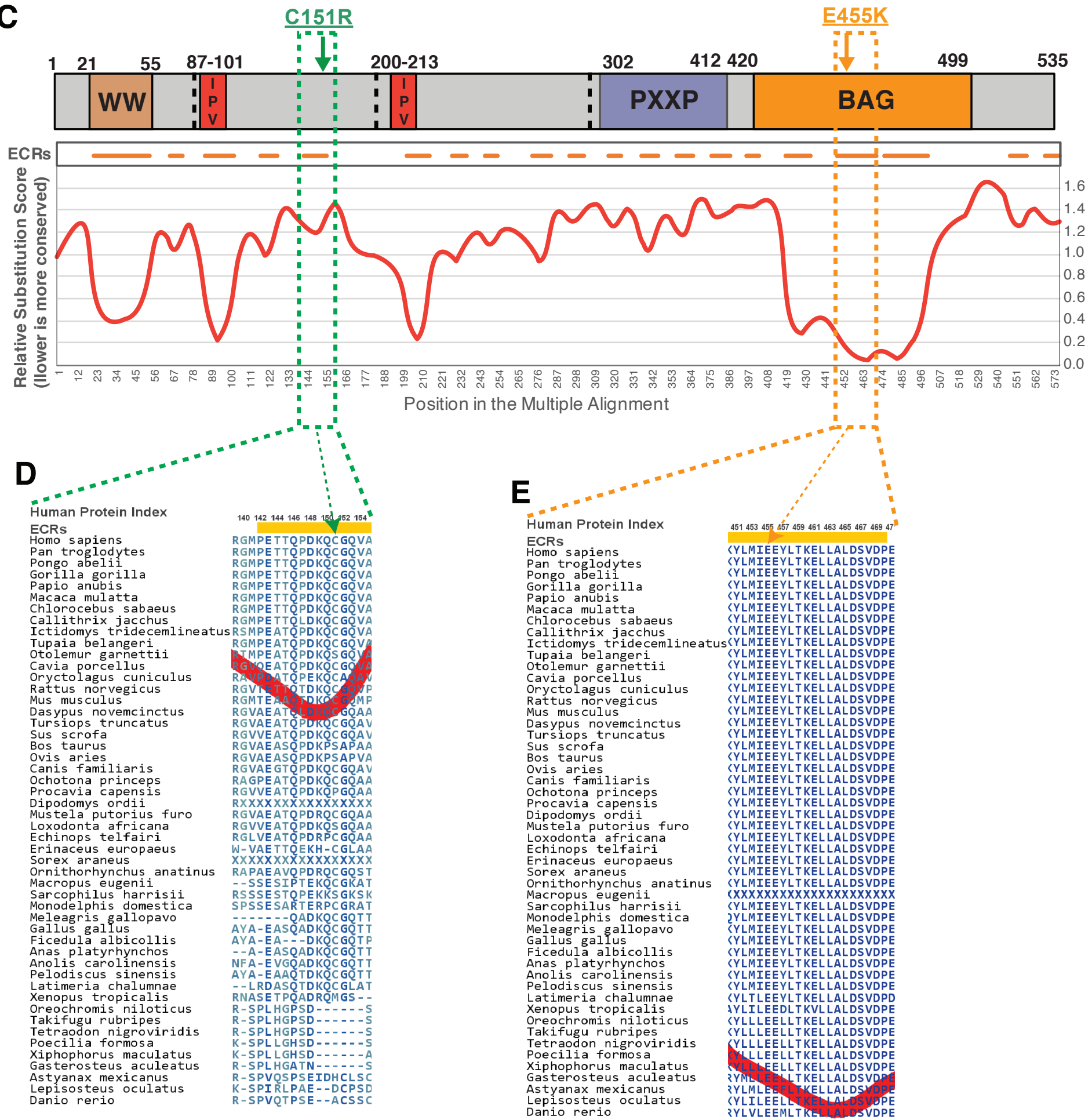
bioRxiv preprint doi: https://doi.org/10.1101/2021.10.06.463213; this version posted October 6, 2021. The copyright holder for this preprint (which was not certified by peer review) is the author/funder, who has granted bioRxiv a license to display the preprint in perpetuity. It is made available under aCC-BY-ND 4.0 International license.

Figure S1. Expanded genetic data graphs for rs2234962, BAG3 ${ }^{\mathrm{C} 151 \mathrm{R}}$. Conservation of residues affected by $B A G 3^{C 151 R}$ and $B A G 3^{E 455 K}$ variants. (A) Zoomed out version of Figure $1 \mathrm{~A}$, showing a window of $200 \mathrm{~KB}$. Dot color indicates type of nucleotide change. (B) Allele frequency map for rs2234962 depicting all 1000 Genomes populations. (C) Top: Diagram of BAG3 domain structure. Bottom: Amino acid conservation plot for matching BAG3 regions.

Decreasing Relative Substitution Score regions (valleys) indicate sequences with high conservation across species and are annotated as Evolutionary Constrained Regions (ECRs). $(\mathrm{D}-\mathrm{E})$ Zoomed in regions for the ECRs around $\mathrm{BAG} 3^{\mathrm{C} 151}(\mathrm{D})$ and $\mathrm{BAG} 3^{\mathrm{E} 455}(\mathrm{E})$. 
bioRxiv preprint doi: https://doi.org/10.1101/2021.10.06.463213; this version posted October 6, 2021. The copyright holder for this preprint

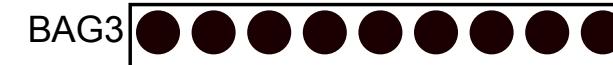
HSPA1A $\bigcirc \bigcirc 0 \bullet$ HSPA8 $\bigcirc \bigcirc \bigcirc \bullet$ HSPA1L O HSPA6 $\bigcirc 000$ HSPA2 $\bigcirc \bigcirc \bigcirc$ HSPA7 $\bigcirc 00$ HSPA9 $O O O O 00$ DNAJC7 0000 DNAJB DNAJB4 DNAJB6 DNAJA1 0 - 0 40

DNAJA2 o $0 \quad 0$

STUB1OOOO 00

MLF2OOOO 00 UBAP2L 0 ○ 0

AMOTO • 0 O

HSPB1

WBP2

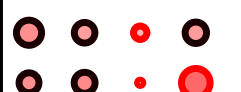

00

CPSF6

$0 \circ 0$

DVL2

PDLIM7

HNRNPUL1

IRS4

-

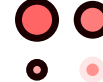

0

0
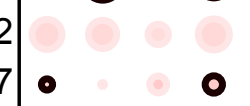

○

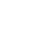

SF1

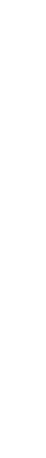

$$
\text { SN }
$$

SNW1

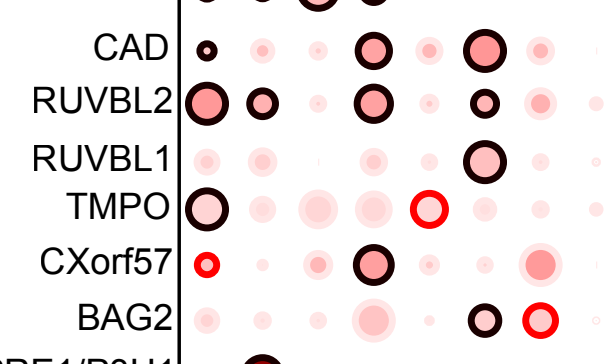

LEPRE1/P3H1

CRTAP

TIMM50

TUBB3

TUBB1

TUBB6

HSPA4

$\mathrm{HSPH} 1$

HSPA4L

POLR2A

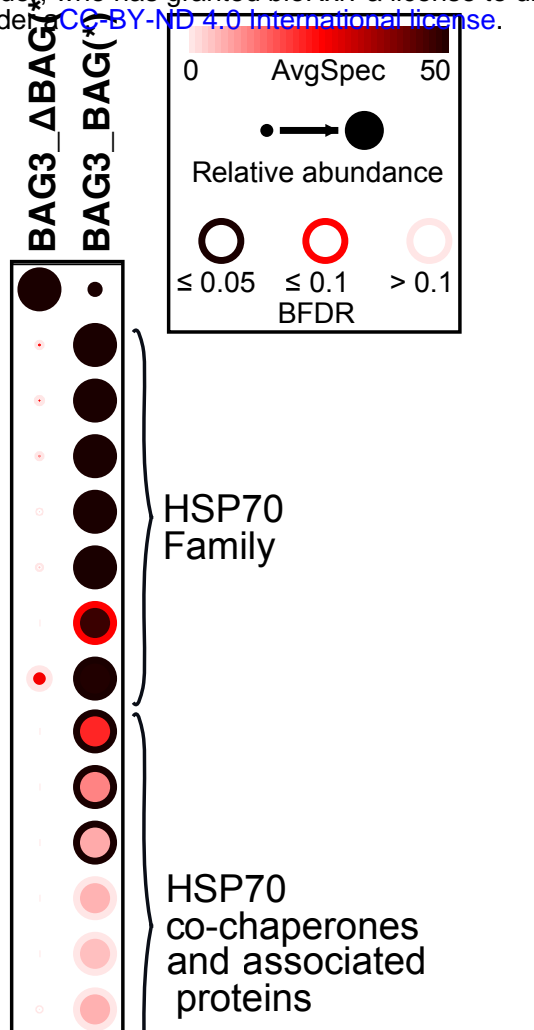

BAG domain independent partners

BAG domain associated partners
Figure S2. Co-precipitation profiles of different BAG3 variants overexpressed in a HEK293 cell background. Dot size represents the amount of co-precipitated protein normalized across variants. Dot color represents absolute protein abundance (spectral counts). Dot rim represents statistical significance. Yellow colored variants are known pathogenic variants associated with DCM. Green variant is putative cardioprotective variant BAG3 $^{\mathrm{C} 151 \mathrm{R}}$. Red variant $\left(B A G 3^{\text {P209L }}\right.$ ) is associated with skeletal myofibrillar myopathy. Black variants are not associated to DCM or any other pathology. Rightmost two columns depict data for BAG3 truncated variant without the BAG domain (BAG3_ $\triangle \mathrm{BAG}$ ) and for the BAG3 protein BAG domain only (BAG3_ $\triangle B A G$ ). For the truncated variants, no normalization by bait levels was performed. $N=5$, BFDR obtained using SAINTexpress (see methods). 
A

B
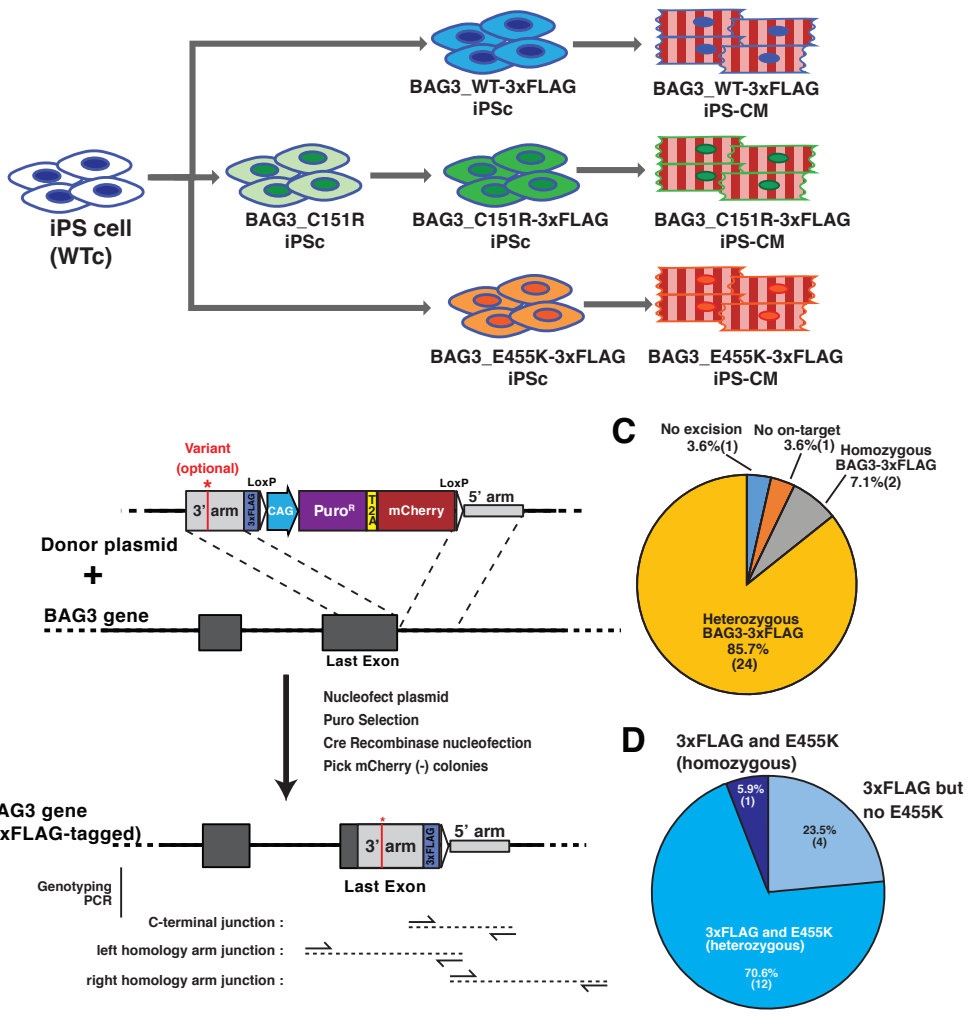

E

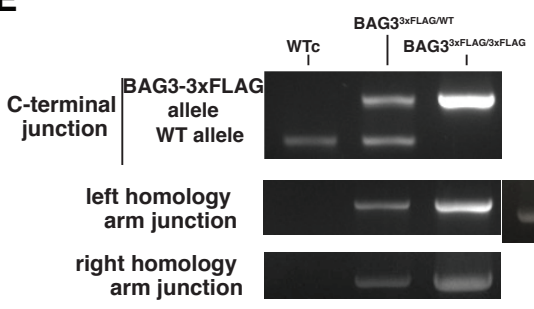

$\mathbf{F}$

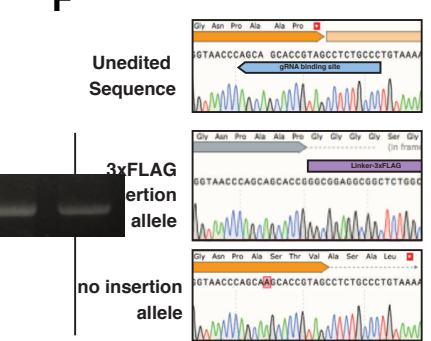

G

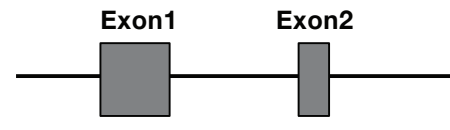

BAG3 locus

H

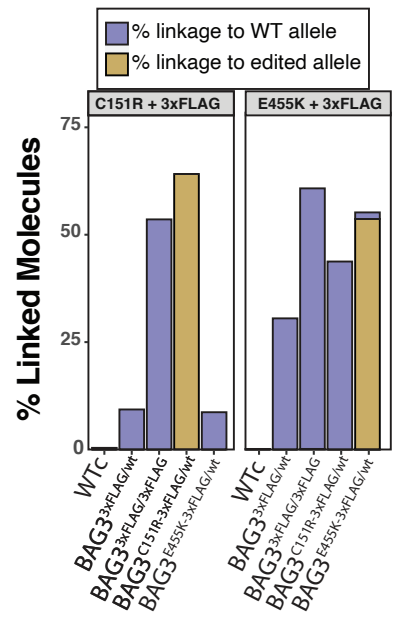

Figure S3. Generation of the isogenic cell lines carrying BAG3 variants and a 3xFLAG epitope tag fusion in the endogenous copy of the BAG3 gene. (A) Workflow for the cell line generation. (B) Strategy for the insertion of a $3 x F L A G$ epitope fusion at the $\mathrm{C}$-terminal of the BAG3 gene. The BAG3 ${ }^{\text {C151R-FLAG }}$ variant was generated using the same process on a preexisting cell line bearing the C151R mutation. To generate the BAG3 ${ }^{\text {E455K-FLAG }}$ cell line, the homology arms were engineered to contain the SNP and insert it during recombination. (C-D) Genotypes of the single-cell clones picked for 3xFLAG insertion $(\mathrm{C})$ and the cosegregation of the BAG3 ${ }^{\mathrm{E} 455 \mathrm{~K}}$ variant (D). (E) Genotyping the products of the 3xFLAG insertion by PCR. (F) Cells with a heterozygous insertion of the 3XFLAG epitope tag also had a SNP in the other allele that extended the BAG3 protein product by 4 amino acids. (G-H) A droplet digital PCR phasing test was used to select clones that contained the desired SNP variants and the 3xFLAG Cterminal sequence in the same allele. The test used different probes $(G)$ to generate an estimate of linked molecules for each cell line and probe combination $(\mathrm{H}$; See Methods for more details) (I) Insertion of the 3xFLAG fusion in the BAG3 gene did not alter the protein levels. $N=3$; one-way ANOVA. 
A

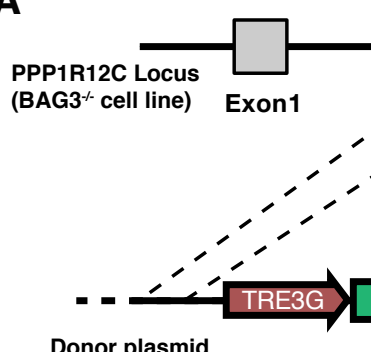

Donor plasmid
Left TALEN IIIIIIIIIIIIIII) IIII)

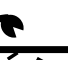

Right TALEN

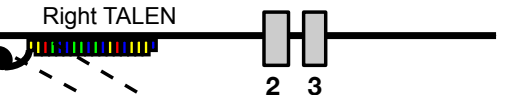

BAG3 cDNA
23
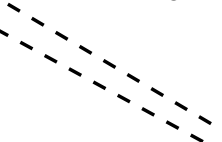

rtTA3G

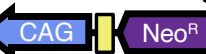

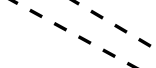

B

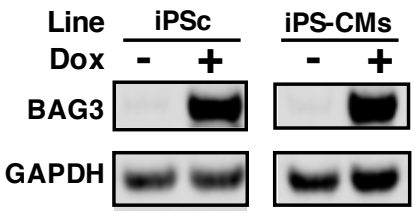

Figure S4. Generation of a cell line with inducible expression of the BAG ${ }^{\mathrm{WT}}$ protein. (A) Diagram of the editing strategy. On a BAG3 ${ }^{-/}$cell background, a doxycycline-activated BAG33xFLAG expression cassette was inserted in the PPP1R12C (AAVS1) safe-harbor locus. (B) Western blot of the BAG3 expression on BAG3 ${ }^{-1-}$ :TetOn-BAG3 ${ }^{\text {WT-3xFLAG }}{ }^{\text {iPSCs }}$ and iPS-CMs with and without Doxycycline addition. 
bioRxiv preprint doi: https://doi.org/10.1101/2021.10.06.463213; this version posted October 6, 2021. The copyright holder for this preprint (which was not certified by peer review) is the author/funder, who has granted bioRxiv a license to display the preprint in perpetuity. It is made

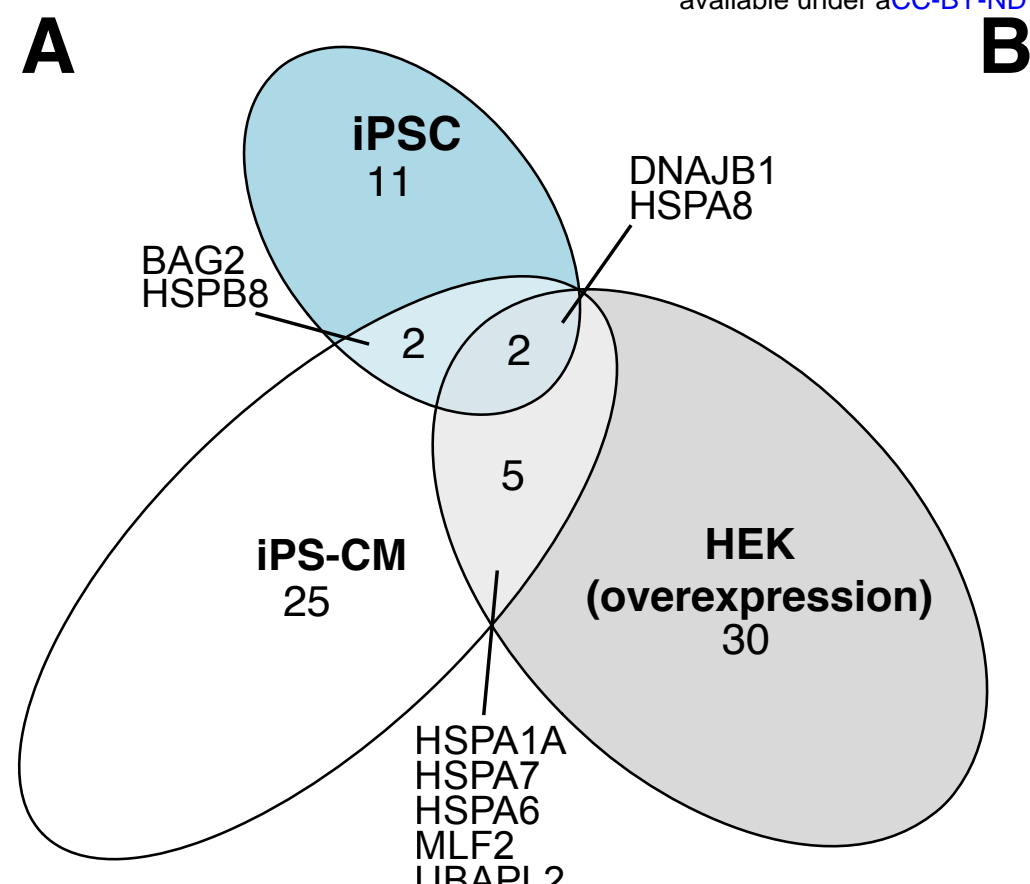
available under aCC-BY-ND 4.0 International license.
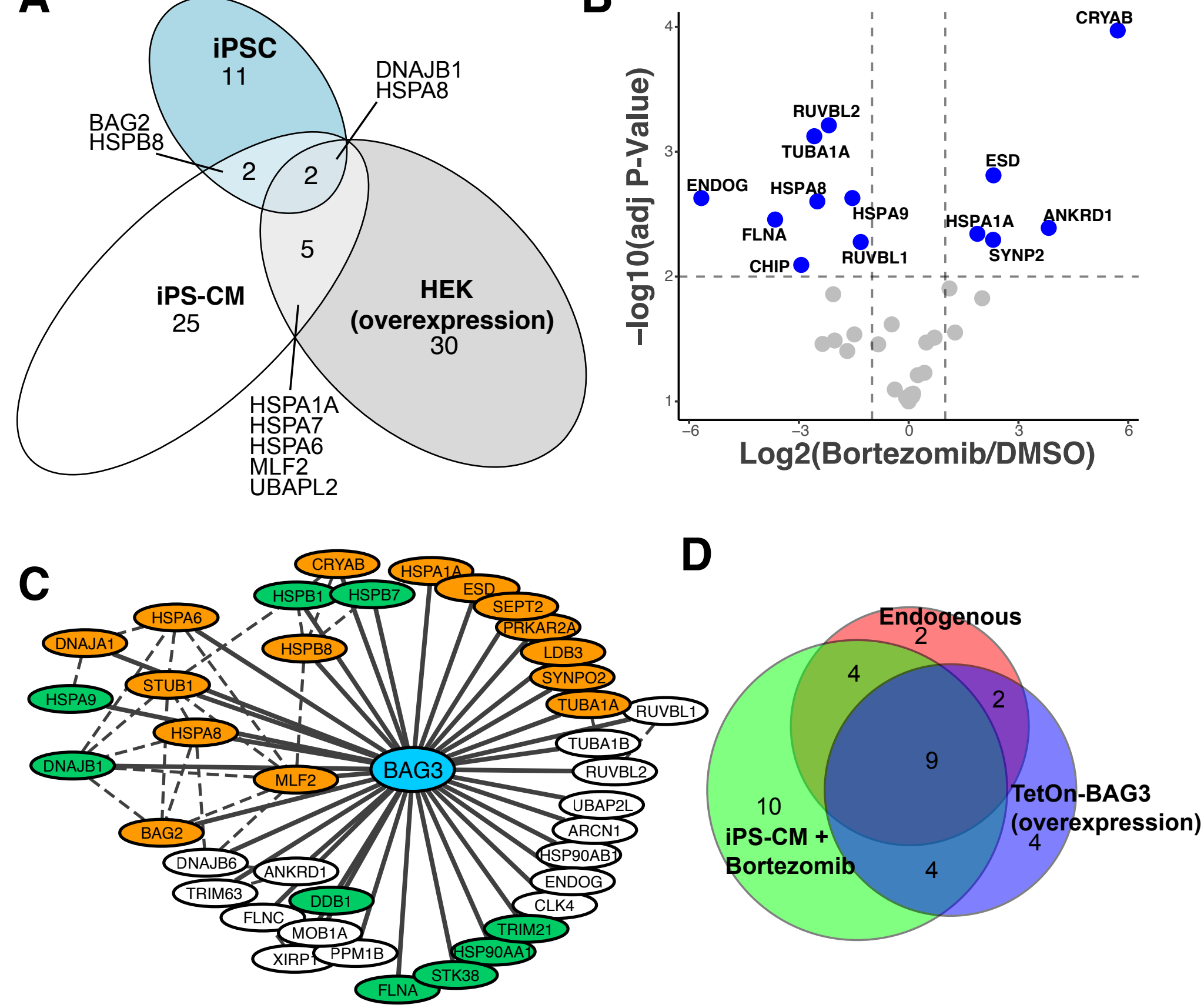

Figure S5. Affinity purification - mass spectrometry characterization of BAG3 binding partners in a cardiomyocyte background. (A) Venn diagram of the high confidence BAG3 ${ }^{\mathrm{WT}}$ protein-protein interactions identified in three cellular backgrounds. HEK293T cells had overexpressed baits, while iPSC have much lower levels of endogenous BAG3 expression than iPS-CM, which could have influenced the results. Each cell type specific dataset was scored separately against its own matched control(s). (B) Volcano plots depicting coprecipitation intensity in BAG3 ${ }^{\mathrm{WT}}$ cardiomyocytes treated with Bortezomib (100nM) relative to DMSO $(1: 10.000)$ for 24 hours. Horizontal dashed line indicates statistical significance threshold (adjusted $p$-value $<0.01$ ) and vertical dashed lines indicate a fold change of 2. $N=4$. (C) Network diagram of the iPS-CM co-precipitation partners identified for BAG3 in this study. Nodes in orange indicate partners that significantly changed when pulling down $B A G 3^{\mathrm{E} 455 \mathrm{~K}}$. Nodes in Green indicate partners that significantly changed when pulling down BAG3 ${ }^{\mathrm{C} 151 \mathrm{R}}$. Dashed lines: known interactions in the iReflndex database. (D) Venn diagram comparing the BAG3 binding partners identified in an iPS-CM background when using endogenous basal levels of expression, an overexpression system, or endogenous expression under proteotoxic stress. The graph highlights the importance of using endogenous expression for accurate characterization of binding partners, and the information gained from using a stress state. 
A

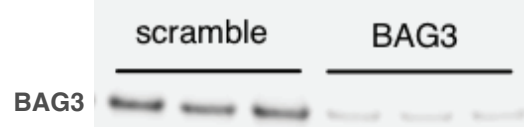

GAPDH

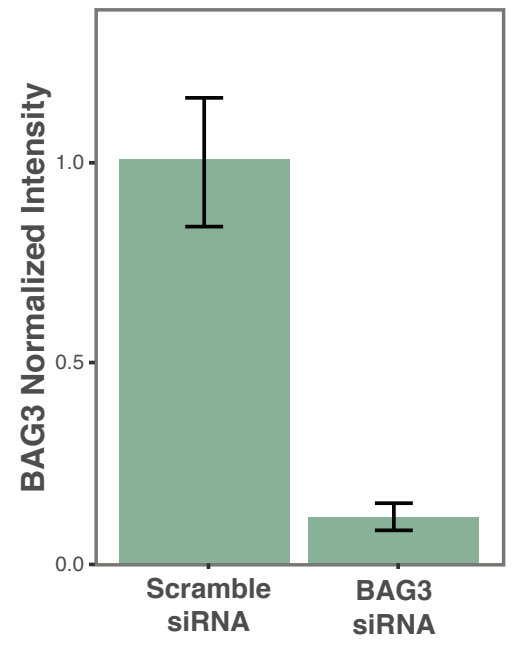

ava Ele under aCC-BMNH-460Binternational license. BAG3
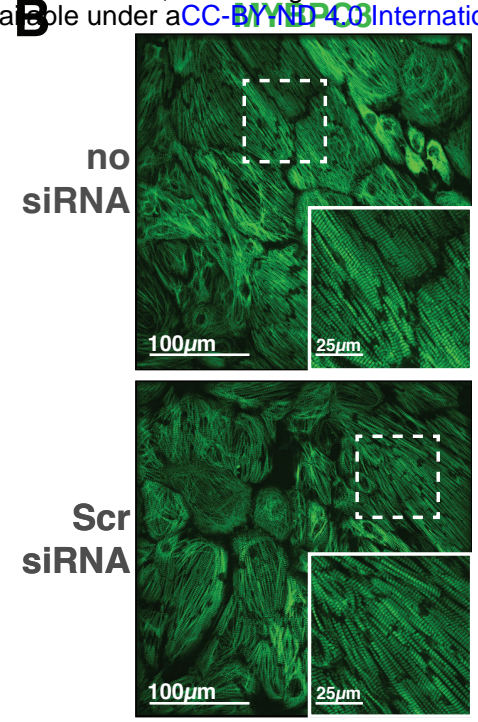

BAG3

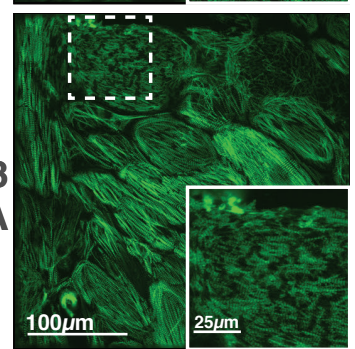

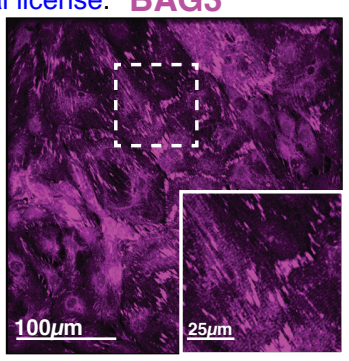
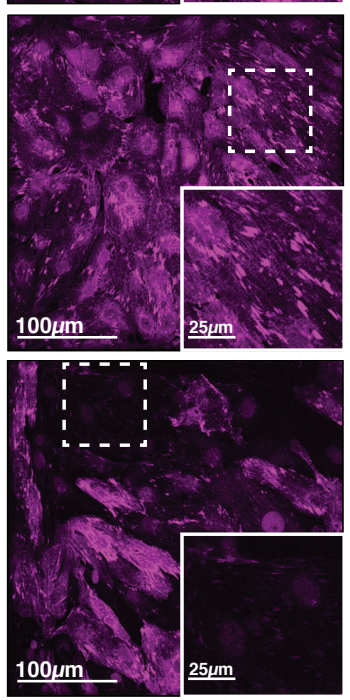
MYBPC3 BAG3 DAPI
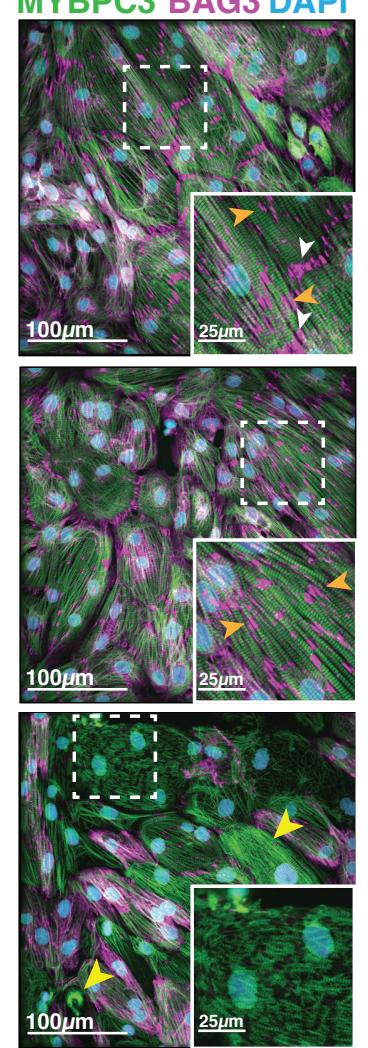

C
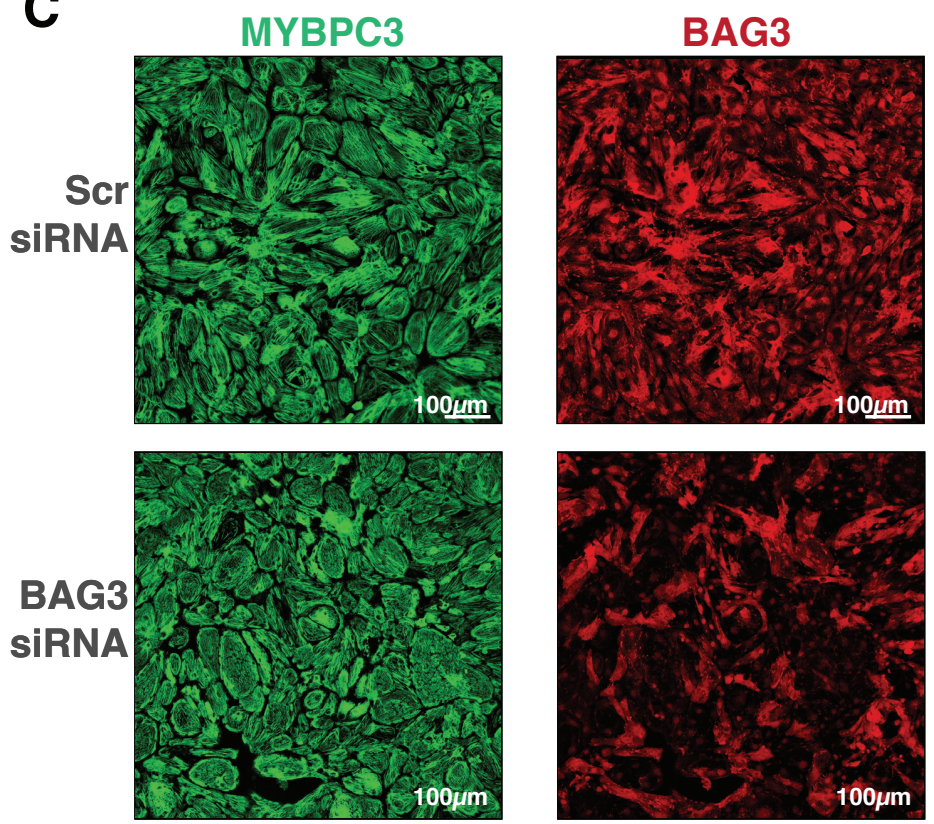
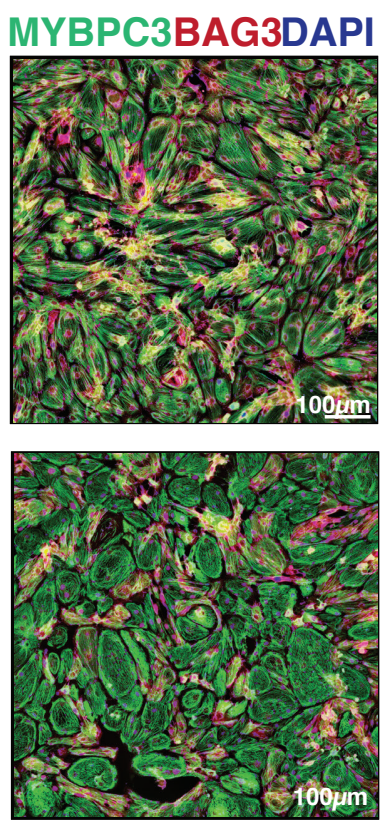

Figure S6. Additional sample micrographs from BAG3 knockdown iPS-CM. (A) BAG3 silencing by siRNA was effective at reducing protein levels ( $85 \%$ reduction). $N=3$. (B) Additional sample micrographs from BAG3and Scr-siRNA-treated iPS-CM, plus a no-siRNA condition. Orange arrowheads: BAG3 accumulation on myofibrillar breaks; white arrowheads: BAG3 accumulation on polar ends of cells; yellow arrowheads: iPS-CM displaying myofibrillar aggregation and collapse. (C) Sample images in the same magnification used for the automated scoring analysis. Lower magnification allowed for faster acquisition and richer features to use directly in the scoring scheme, but higher magnification images were used elsewhere in this manuscript for easier viewing. 
bioRxiv preprint doi: https://doi.org/10.1101/2021.10.06.463213; this version posted October 6, 2021. The copyright holder for this preprint (which was not certified by peer review) is the author/funder, who has granted bioRxiv a license to display the preprint in perpetuity. It is made

A

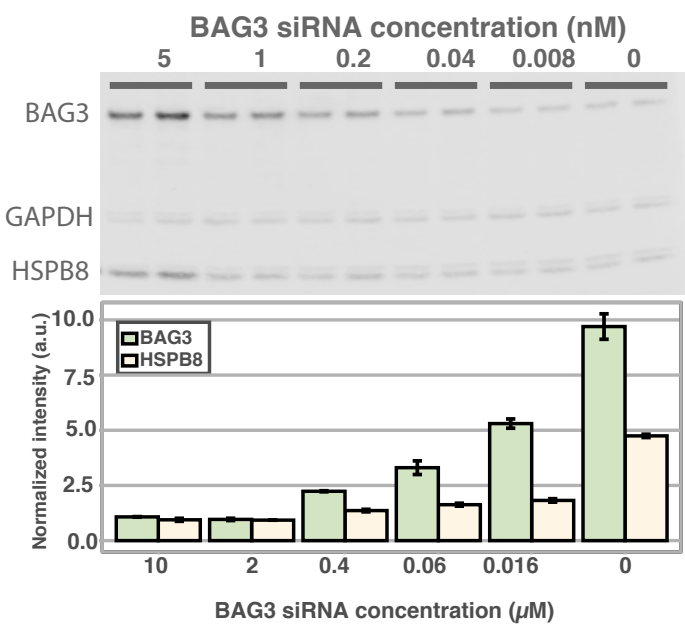

C

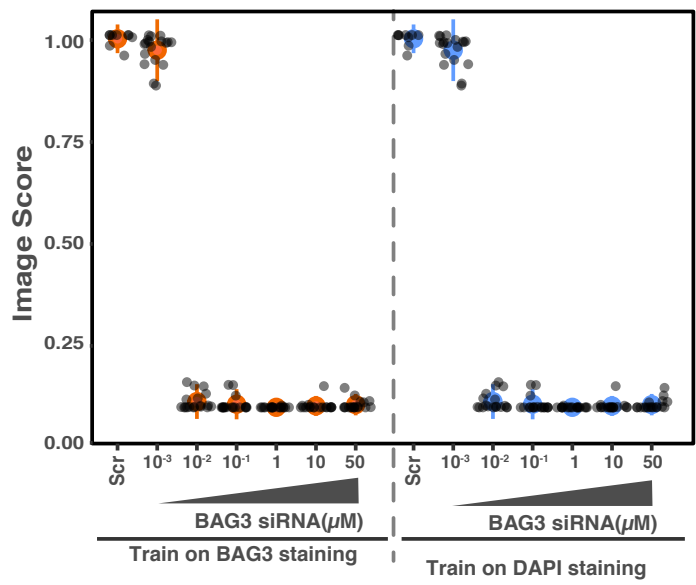

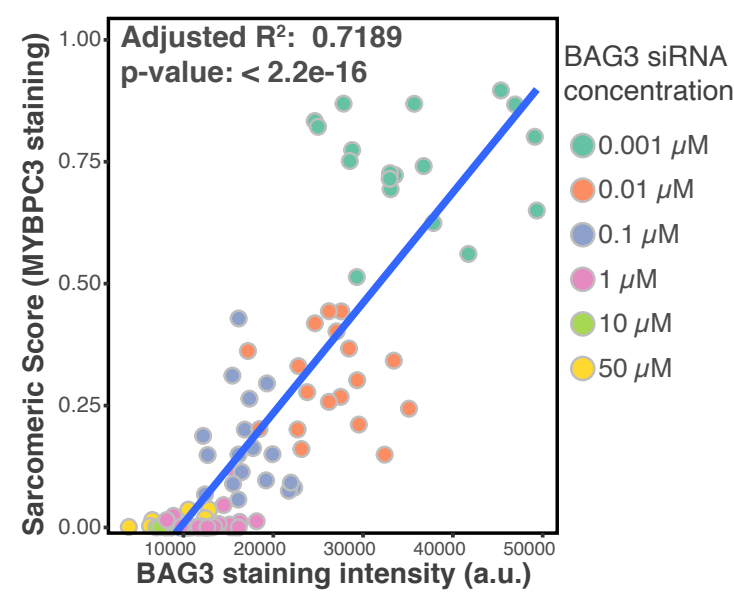

D

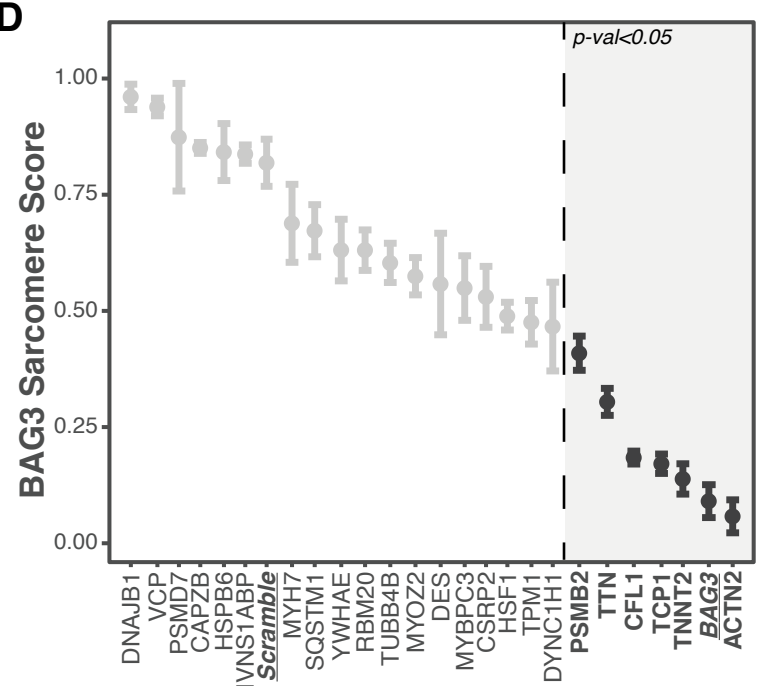

$\mathbf{E}$
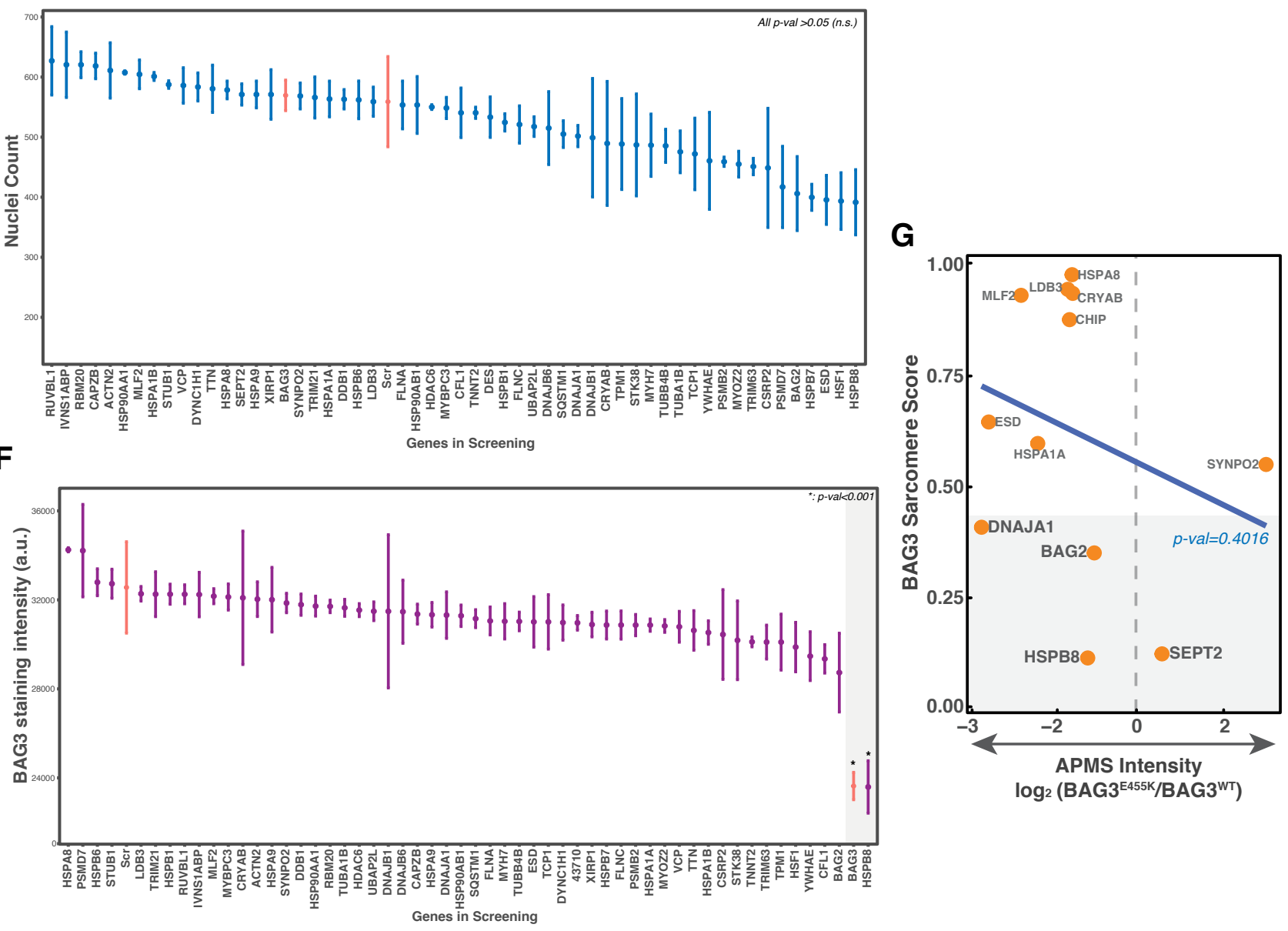
Figure S7. Quality control and additional data from the siRNA knockdown-myofibrillar scoring workflow. (A) Western blot demonstrating the titration of BAG3 siRNA results in decreasing cellular BAG3 protein levels. Shown at the bottom of the western blot are levels of HSPB8, which are also affected by BAG3 knockdown. $N=2$. (B) BAG3 sarcomere score inversely correlates with BAG3 protein levels. $N=18$ images. (C) Plot of the image scores that result from training based on BAG3 or DAPI staining. These stains do not display the same dynamic range as scoring based on myofibrillar (MYBPC3) staining. $N=9$ for scramble; 18 for the rest. (D) BAG3 Sarcomere Score for the knockdown of selected factors that were not identified in our AP-MS coprecipitation studies. Dots represent mean of 3 replicates from separate wells, each being the median score of 9 images from the same well. Error bars: SEM. P-val cutoff: 0.05 using a one-way ANOVA with post-hoc Dunnett test. (E-F) Plot of the nuclei count $(E)$ and BAG3 staining $(F)$ intensities for the gene knockdowns used in the siRNAmyofibrillar scoring analyses. Dots represent mean of 3 replicates from separate wells, each being the median score of 9 images from the same well. Error bars: SEM. P-val cutoff: 0.05 using a one-way ANOVA with post-hoc Dunnett test. (G) Plotting of the APMS intensity ratio for $B A G 3^{\mathrm{E} 455 \mathrm{~K}}$ differential interactors and their BAG3 Sarcomere Score. There is no statistically significant correlation. P-value obtained fitting a linear model. Pearson's product-moment correlation: -0.27 . 
A
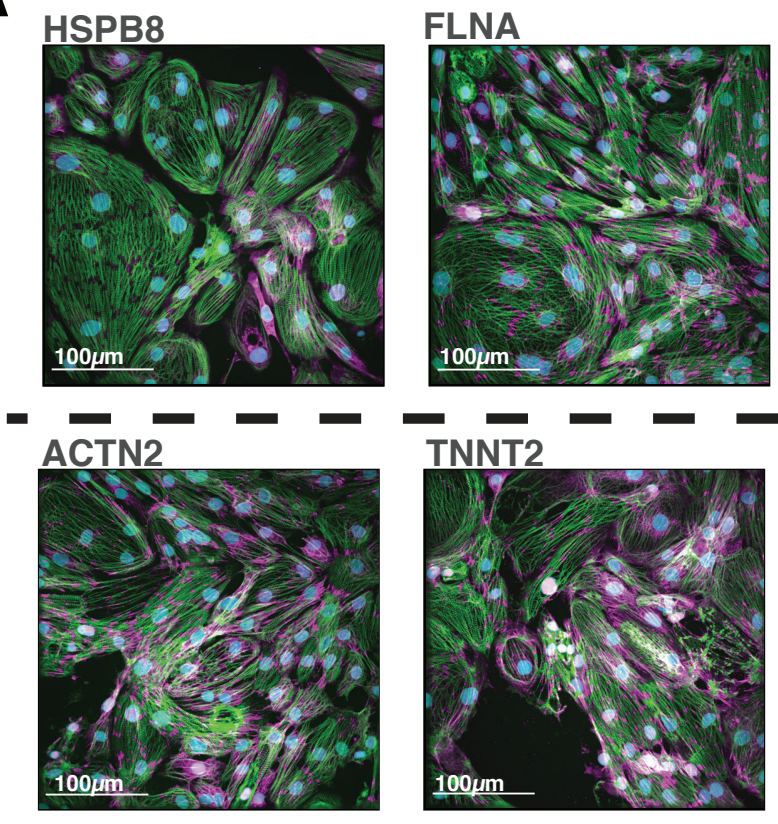
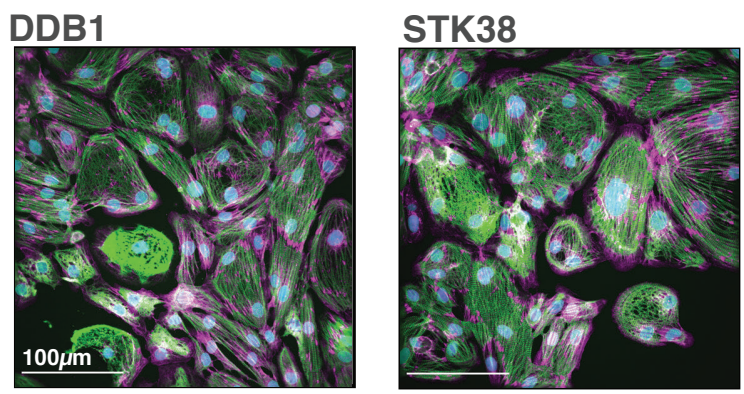

HSPB7
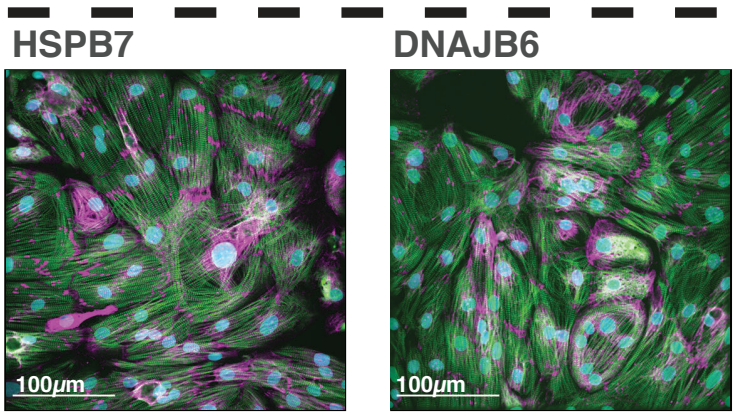

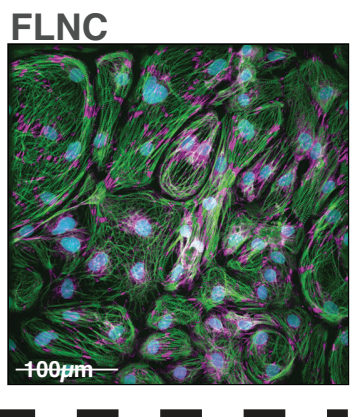

Figure S8. Sample images from selected siRNA knockdowns. HSPB8 knockdown was the only knockdown to significantly reduce BAG3 levels. FLNA, DDB1 and STK38 are BAG3 ${ }^{\mathrm{C} 151 \mathrm{R}}$ differential interactors whose knockdown resulted in sarcomere scores similar to BAG3 knockdown. ACTN2 and TNNT2 are well known sarcomere components that display low sarcomere scores similar to BAG3 knockdown, possibly due to reduced sarcomeric density and increased disarray. HSPB7 and DNAJB6 knockdowns displayed high sarcomere scores (similar to Scramble control). For all images, scale bar $=100$ $\mu \mathrm{M}$. Magenta: BAG3; Green: MYBPC3; Cyan: DAPI. 
bioRxiv preprint doi: https://doi.org/10.1101/2021.10.06.463213; this version posted October 6, 2021. The copyright holder for this preprint (which was not certified by peer review) is the author/funder, who has granted bioRxiv a license to display the preprint in perpetuity. It is made
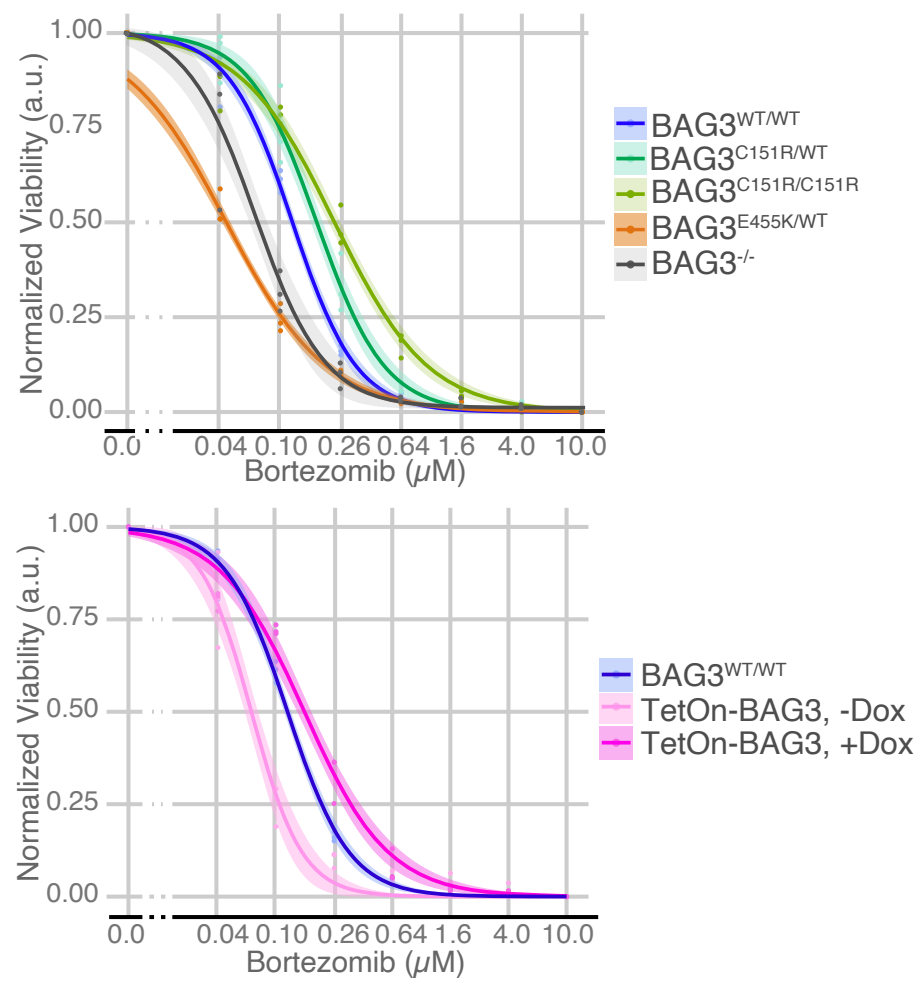

B International license.

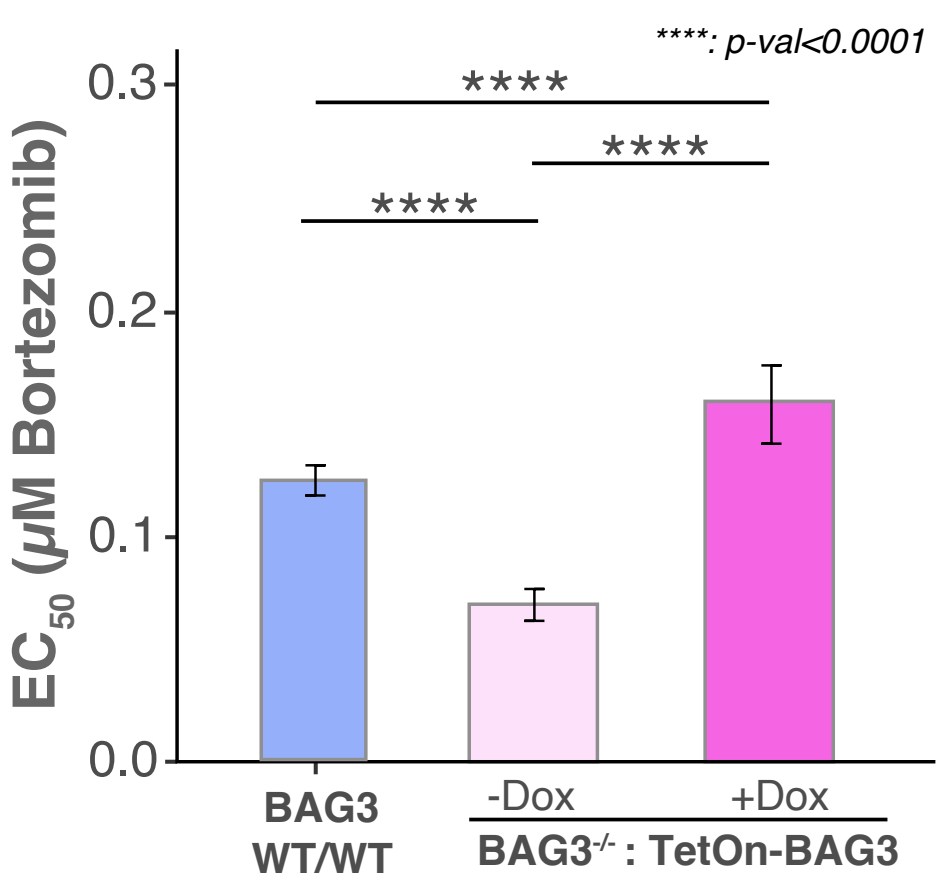

C

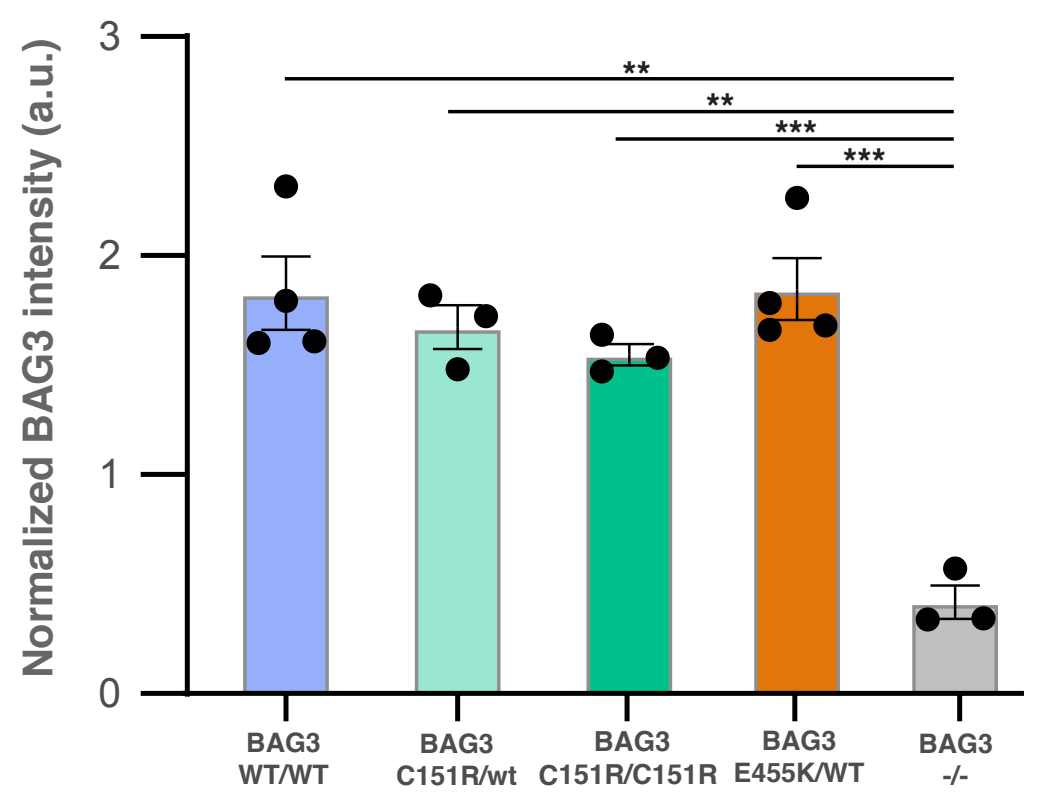

Figure S9. BAG3 overexpression rescues bortezomib sensitivity phenotype in BAG3 ${ }^{-1-}$ cells, and $B A G 3^{\mathrm{C} 151 \mathrm{R}}$ and $B A G 3^{\mathrm{E} 455 \mathrm{~K}}$ do not change BAG3 protein levels in iPS-CMs. (A) Bortezomib dose-response curves for the data used for $\mathrm{EC}_{50}$ calculations. (B) Calculated $\mathrm{EC}_{50}$ and $95 \%$ confidence intervals for Bortezomib in control (WT/WT), and BAG3 ${ }^{-1 /}$ iPS-CM with and without BAG3 overexpression. $N=3$. ${ }^{* * *}$ : $P$-value $<0.0001$ and *: $p$-value $<0.5$ using one-way ANOVA with post-hoc Zidak correction. (C) Capillary immunoassay (Simple Western) quantification of BAG3 protein levels in IPS-CM differentiated from iPSCs heterozygous or homozygous for the indicated BAG3 alleles. $N=3 .{ }^{* *}$ : $p$-value $<0.01 ;{ }^{* * *}$ : $p$-value $<0.001$. Oneway ANOVA with post-hoc Tukey multiple comparisons test. 